ARTICLE

DOI: $10.1038 / s 41467-018-05663-x$

\title{
Variation in bradyrhizobial NopP effector determines symbiotic incompatibility with Rj2-soybeans via effector-triggered immunity
}

\author{
Masayuki Sugawara', Satoko Takahashi ${ }^{1}$, Yosuke Umehara², Hiroya Iwano', Hirohito Tsurumaru ${ }^{3}$ \\ Haruka Odake ${ }^{1}$, Yuta Suzuki ${ }^{1}$, Hitoshi Kondo ${ }^{1}$, Yuki Konno ${ }^{1}$, Takeo Yamakawa ${ }^{4}$, Shusei Sato ${ }^{1}$, Hisayuki Mitsui ${ }^{1} \&$ \\ Kiwamu Minamisawa ${ }^{1}$
}

Genotype-specific incompatibility in legume-rhizobium symbiosis has been suggested to be controlled by effector-triggered immunity underlying pathogenic host-bacteria interactions. However, the rhizobial determinant interacting with the host resistance protein (e.g., Rj2) and the molecular mechanism of symbiotic incompatibility remain unclear. Using natural mutants of Bradyrhizobium diazoefficiens USDA 122, we identified a type III-secretory protein NopP as the determinant of symbiotic incompatibility with Rj2-soybean. The analysis of nopP mutations and variants in a culture collection reveal that three amino acid residues (R60, R67, and $\mathrm{H} 173$ ) in NopP are required for Rj2-mediated incompatibility. Complementation of rj2-soybean by the Rj2 allele confers the incompatibility induced by USDA 122-type NopP. In response to incompatible strains, Rj2-soybean plants activate defense marker gene $P R-2$ and suppress infection thread number at 2 days after inoculation. These results suggest that Rj2soybeans monitor the specific variants of NopP and reject bradyrhizobial infection via effector-triggered immunity mediated by Rj2 protein.

\footnotetext{
${ }^{1}$ Graduate School of Life Sciences, Tohoku University, 2-1-1 Katahira, Aoba-ku, Sendai, Miyagi 980-8577, Japan. ${ }^{2}$ Institute of Agrobiological Sciences, National Agriculture and Food Research Organization, 2-1-2 Kannondai, Tsukuba, Ibaraki 305-8602, Japan. ${ }^{3}$ Faculty of Agriculture, Kagoshima University, 1-21-24 Korimoto, Kagoshima 890-0065, Japan. ${ }^{4}$ Faculty of Agriculture, Graduate School of Bioresource and Bioenvironmental Sciences, Kyushu University, 6-10-1 Hakozaki, Higashi-ku, Fukuoka 812-8581, Japan. Correspondence and requests for materials should be addressed to

M.S. (email: msugawara@ige.tohoku.ac.jp)
} 
T he type III secretion system (T3SS) is common to bacterial pathogens of plants and animals ${ }^{1,2}$. It delivers effector proteins directly into the host cells ${ }^{1,2}$ and helps the pathogen to survive and to escape the immune response activated by microbe/pathogen-associated molecular patterns. To counter the virulence functions of effectors, plants have evolved resistance $(R)$ genes encoding nucleotide-binding site/leucine-rich repeat (NBS-LRR) proteins that often directly or indirectly recognize specific effectors and mount a strong immune response called effector-triggered immunity (ETI) ${ }^{3}$.

Rhizobia symbiotically reduce atmospheric dinitrogen in root nodules to ammonia that is utilized by the host legumes, resulting in improved plant growth for sustainable agriculture $^{4,5}$. During the establishment of symbiosis, rhizobia generally produce lipochitin oligosaccharides, called Nod factors (NFs), in response to plant flavonoid compounds to activate host signaling, leading to rhizobial infection and nodule organogenesis ${ }^{6}$. Symbiotic specificity between legumes and rhizobia is initially determined by the structure of NFs and host NF receptor kinases (e.g., NFR1 and NFR5 in Lotus japonicus) 7,8 . However, many rhizobia have genes encoding T3SS structural components ( $r h c$ genes) such as pathogens and the effector proteins often stimulate the host immune system and alter the host range $\mathrm{e}^{9-12}$.

A number of rhizobial T3SS effector proteins have been identified as nodulation outer proteins (Nops) ${ }^{10,13}$. Flavonoids derived from host plants activate a transcriptional regulator NodD, which induces the expression of $t t s I$, a gene for a positive transcriptional regulator of the $r h c$ and nop genes ${ }^{14,15}$. Several Nops suppress plant defense reactions and hence promote nitrogen-fixing nodule symbiosis ${ }^{16}$. By genomic analysis, more than 30 genes in Bradyrhizobium and approximately 15 genes in Sinorhizobium fredii have been predicted to encode T3SS effectors ${ }^{17}$, but their symbiotic and biochemical functions have not been fully elucidated.

Soybean [Glycine $\max$ (L.) Merr.] is a major leguminous crop worldwide. The plants form root nodules upon infection with nitrogen-fixing bradyrhizobia, such as Bradyrhizobium diazoefficiens, Bradyrhizobium japonicum, and Bradyrhizobium elkanii ${ }^{18,19}$. Several dominant genes (Rj2, Rj3, Rj4, and $R f g 1$ ) in soybeans restrict nodulation with specific rhizobial strains ${ }^{20}$. Among them, Rj2-genotype soybeans restrict nodulation with specific Bradyrhizobium strains, including B. diazoefficiens USDA $122^{21,22}$. Positional cloning has revealed that the Rj2 gene encodes a typical $\mathrm{R}$ protein of the Toll-interleukin receptor/NBS/LRR (TIR-NBS-LRR) class ${ }^{23}$. This finding indicates that symbiotic incompatibility between USDA 122 and $R j 2$-soybean plants may be controlled by ETI. The inactivation of the T3SS in $B$. diazoefficiens USDA 122 restores its nodulation capability on the Rj2-soybean cultivar Hardee $^{24}$. The deduced amino acid sequences of proteins encoded by the rhc and ttsI genes are identical between $B$. diazoefficiens USDA 122 and the compatible strain $B$. diazoefficiens USDA $110^{\mathrm{T}}$, suggesting that a T3SS-dependent effector protein in USDA 122 block nodulation with Rj2-soybean plants ${ }^{24}$. However, the T3SS effector and the molecular mechanism of this genotype-specific incompatibility have been unknown.

Here we have obtained spontaneous mutants of $B$. diazoefficiens USDA 122 that overcome Rj2-mediated incompatibility and identified NopP as a causal effector of this incompatibility. We also reveal that, among different amino acid residues of NopP in strain USDA 122 and a compatible strain USDA $110^{\mathrm{T}}$, three amino acid residues (R60, R67, and $\mathrm{H} 173$ ) are required for inducing this symbiotic incompatibility via ETI mediated by $\mathrm{Rj} 2$ protein.

\section{Results}

Nodulation phenotypes of USDA 122 and its T3SS mutants. The Rj2-genotype soybean cultivar Hardee restricts nodulation with wild-type $B$. diazoefficiens USDA 122 but not with USDA 122 carrying mutated genes for a T3SS structural component $(122 \Omega r h c J)$ and a positive transcriptional regulator of T3SSrelated genes $(122 \Omega t t s I)^{24}$. Consistent with this report, Hardee plants inoculated with $122 \Omega r h c J$ and $122 \Omega$ ttsI formed significantly more nodules than those inoculated with wild-type USDA 122; the number of nodules induced by USDA $110^{\mathrm{T}}$ was comparable to that of $122 \Omega r h c J$ and $122 \Omega$ ttsI (Fig. 1a). As the plants inoculated with $122 \Omega r h c J$ or $122 \Omega$ ttsI grew well and had green leaves in nitrogen-free medium (Fig. 1b), the corresponding nodules apparently fixed $\mathrm{N}_{2}$.

Wild-type USDA 122 formed no or only a single nodule at a low frequency and its nodulation phenotype was clearly distinct from those of $122 \Omega \mathrm{rhcJ}$ and $122 \Omega \mathrm{ttsI}$ (Fig. 1a, c, d). We considered two explanations for the sporadic nodulation on Hardee roots: (i) a few cells of wild-type USDA 122 passed through the barrier of Rj2-mediated incompatibility or (ii) the single nodules were formed by spontaneous USDA 122 mutants with defects in genes responsible for Rj2-mediated incompatibility.

Spontaneous mutants overcoming Rj2-mediated incompatibility. We inoculated 26 Hardee plants with wild-type USDA 122, purified eight isolates (W1-1a, W3-1a, W3-2a, W81a, W8-1b, W9-1a, W9-1b, and W9-3a) from eight independent nodules, and re-inoculated the isolates onto Hardee. All isolates consistently formed more nodules on Hardee roots $\left(\mathrm{Nod}^{+}\right.$phenotype) than did USDA 122 (Fig. 2a, b), clearly showing that they were all spontaneous mutants of USDA 122 with defects in genes necessary to trigger Rj2-mediated incompatibility. However, nitrogen fixation phenotypes differed among the eight isolates, as indicated by acetylene reduction assay (Fig. 2a) and leaf color (Fig. 2b). Strains W3-1a, W9-1a, and W9-1b elicited normal nitrogen-fixing nodules ( $\mathrm{Nod}^{+} / \mathrm{Fix}^{+}$phenotype), whereas $\mathrm{W} 1-1 \mathrm{a}$, W3-2a, W8-1a, W8-1b, and W9-3a showed no nitrogen fixation activity ( $\mathrm{Nod}^{+} / \mathrm{Fix}^{-}$phenotype).

To search for the mutations, we mapped MiSeq reads of the eight mutants on the complete genome sequence of USDA $122^{25}$. In the genomes of the three $\mathrm{Nod}^{+} / \mathrm{Fix}^{+}$mutants, we identified several single-nucleotide polymorphisms (SNPs) and insertions of the insertion sequence (IS) elements ISRj1, ISRj2, and ISBdi2 (Supplementary Table 1). W3-1a and W9-1a had mutations in the nopP gene within the nif gene cluster in a symbiosis island, caused by different IS element insertions (Fig. 2c, Supplementary Fig. 1). As nopP encodes a T3SS effector ${ }^{26,27}$, we considered it a candidate gene for Rj2 symbiotic incompatibility. On the other hand, W9-1b had a SNP within the BD122_09230 locus in a gene cluster including the gene encoding the T3SS structural component $r h c V$ (Fig. 2c), suggesting that this mutant might lack a functional T3SS.

In the genomes of the five $\mathrm{Nod}^{+} / \mathrm{Fix}^{-}$strains, we found ISRj1and ISRj2-mediated deletions that included both nif and $r h c$ gene clusters (Fig. 2c, Supplementary Figs. 2-5). As the nif genes are required for nitrogen fixation, these mutants showed the Fix ${ }^{-}$ phenotype in addition to the $\mathrm{Nod}^{+}$phenotype caused by the lack of the $r h c$ genes, which are required for T3SS function.

Functional validation of nopP on symbiotic incompatibility. $n o p P$ is located in the center of the nif gene cluster (Fig. 3a), and a cis element motif (tts-box) is present upstream $(-100$ to -63$)$ of nopP (Fig. 3b). Genistein, a flavonoid inducer of T3SS genes in bradyrhizobia, greatly increased NopP protein secretion via T3SS 
a

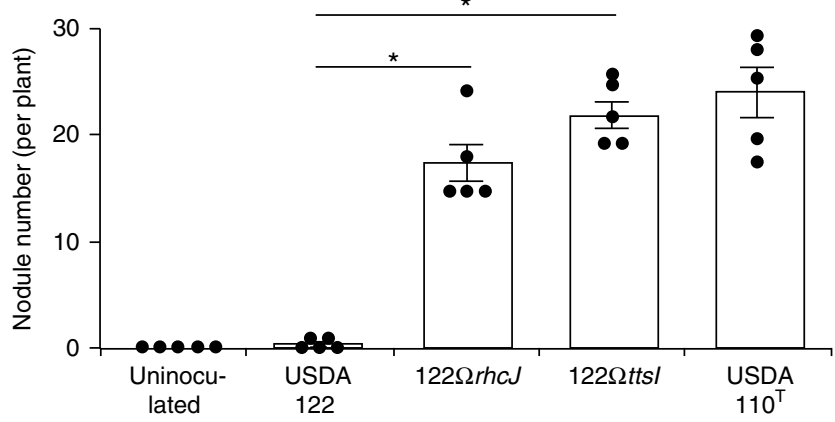

b
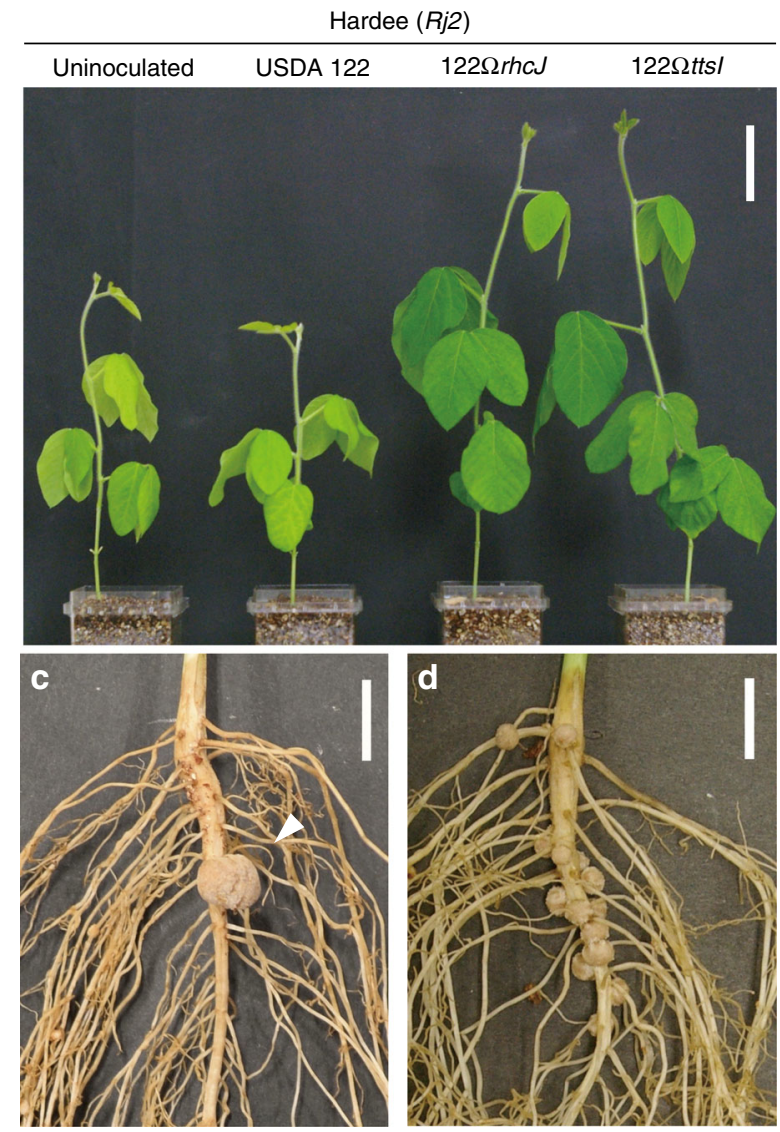

Fig. 1 Symbiotic phenotypes of Hardee (Rj2) inoculated with B. diazoefficiens strains. a Number of nodules formed on roots at 28 days after rhizobial inoculation. No experimental repeat was conducted with the same comparison. Error bars show SEM $(n=5)$. *Significant difference from USDA 122 ( $P<0.01$ by Dunnett's multiple comparison test). b Shoots 28 days after inoculation with the indicated strains. Scale bar, $5 \mathrm{~cm}$. c Roots of plants inoculated with USDA 122. The arrow indicates a single sporadic nodule; normal nodules were rarely formed by USDA 122 . Scale bar, $1 \mathrm{~cm}$. d Roots of plants inoculated with $122 \Omega \mathrm{rhcJ}$. Scale bar, $1 \mathrm{~cm}$

in USDA 122 but its secretion was not detected in $122 \Omega \mathrm{rhc}$ (Fig. 3c). These results indicate that nopP encodes a T3SS effector protein.

To examine whether NopP induces symbiotic incompatibility with Rj2-soybeans, we constructed an in-frame nopP deletion mutant $122 \Delta n o p P$ (Fig. 3a) and inoculated it onto Hardee. Plants inoculated with $122 \Delta n o p P$ formed significantly more nodules than plants inoculated with wild-type USDA 122 (Fig. 3d, e). The symbiotic phenotype of $122 \Delta$ nopP was similar to that of a T3SS mutant $(122 \Omega r h c J)$ in terms of nodulation and nitrogen fixation (Fig. 3d-f). The efficient symbiotic phenotype with $122 \Delta n o p P$ was also observed in other Rj2-soybean cultivars, CNS and IAC-2 (Supplementary Fig. 6a-d). In an rj2-soybean cultivar Lee, the symbiotic phenotypes were not different between inoculations of USDA 122 and 122 $\Delta$ nopP (Supplementary Fig. 6e, f). These results clearly indicate that NopP effector in $B$. diazoefficiens USDA 122 is necessary to trigger $R j 2$ symbiotic incompatibility.

nopP allele swapping between USDA 122 and USDA $110^{\mathrm{T}}$. Although NopP of USDA 122 is a T3SS effector inducing Rj2 symbiotic incompatibility, the nop $P$ gene is also present in $B$. diazoefficiens USDA $110^{\mathrm{T}}$ (Supplementary Fig. 7a) but does not induce Rj2-mediated incompatibility. Moreover, the NopP protein was secreted from USDA $110^{\mathrm{T}}$ cells when genistein is added to culture medium (Fig. 4a) via T3SS ${ }^{27}$, suggesting that symbiotic compatibility between bradyrhizobia and Rj2-soybeans is not determined by secretion of NopP but by its structure.

An alignment of NopP amino acid sequences (277 aa) showed substitutions of four amino acid residues between USDA 122 and $110^{\mathrm{T}}$ (Fig. 4b, Supplementary Fig. 7b). To examine whether the difference determines symbiotic compatibility, we swapped the nopP genes between USDA 122 and $110^{\mathrm{T}}$. A USDA 122 derivative carrying USDA 110 -type nopP $\left(122 n o p P_{110}\right)$ formed significantly more nodules on Hardee than did wild-type USDA 122 and the leaves of $122 n o p P_{110}$ were green, indicating nitrogen-fixing activity of the nodules (Fig. $4 \mathrm{c}, \mathrm{d}$ ). A USDA $110^{\mathrm{T}}$ derivative carrying USDA 122-type nopP (110nop $\left.P_{122}\right)$ showed a nodulation-deficient phenotype similar to that of wild-type USDA 122 (Fig. 4c, d). 110nop $P_{122}$ secreted the NopP protein as well as the wild-type USDA $110^{\mathrm{T}}$ (Fig. 4a), suggesting that substitutions of NopP on the amino acid residues at positions 60 , 67,173 , and 271 does not affect the ability of the bacteria to deliver the protein. These results indicate that the difference of four amino acid residues in NopP between USDA 122 and $110^{\mathrm{T}}$ determines Rj2 symbiotic incompatibility.

To examine when $R j 2$-soybeans block the infection of USDA 122 , we microscopically observed early infection events (Supplementary Fig. 8). The number of infection thread (IT) formed on the main roots inoculated with incompatible strains (USDA 122 or 110 nop $P_{122}$ ) was significantly less than those with compatible strains $\left(122\right.$ nop $P_{110}$ or USDA $\left.110^{\mathrm{T}}\right)$ at 2 days after inoculation (DAI) (Fig. 4e). These results suggest that Rj2-soybeans mainly block the incompatible rhizobia at a step before IT formation.

NopP-mediated symbiotic incompatibility through $\mathbf{R j 2}$ protein. The host $\mathrm{Rj} 2$ protein is a TIR-NBS-LRR class of $\mathrm{R}$ protein, and its E452 and I490 residues located between the NBS and LRR domains are reportedly required for incompatibility with USDA $122^{23}$. To investigate the involvement of $\mathrm{Rj} 2$ in NopP-mediated symbiotic incompatibility, we produced transgenic soybean cultivar Lee ( $r j 2)$ plants complemented with the complementary DNA of Rj2 (Hardee) or rj2 (Hardee, E452K, I490R) and evaluated their nodulation phenotypes by inoculating them with USDA 122 and 122 nop $P_{110}$. As hairy root transformation was conducted without antibiotic selection, the resulting hairy roots were either transgenic or wild type, which could be readily distinguished by examining the presence of green fluorescent protein (GFP) encoded in the used binary vector ${ }^{28}$.

When Rj2-transformed roots were inoculated with USDA 122, nodules were rarely observed on transgenic hairy roots (Fig. 5a, b). In contrast, when they were inoculated with 122 nop $P_{110}$, nodule formation was observed (Fig. 5c, d). Roots transformed with $r j 2$ or empty vector formed nodules regardless of whether it was infected with USDA 122 or $122 n o p P_{110}$ (Fig. 5e 

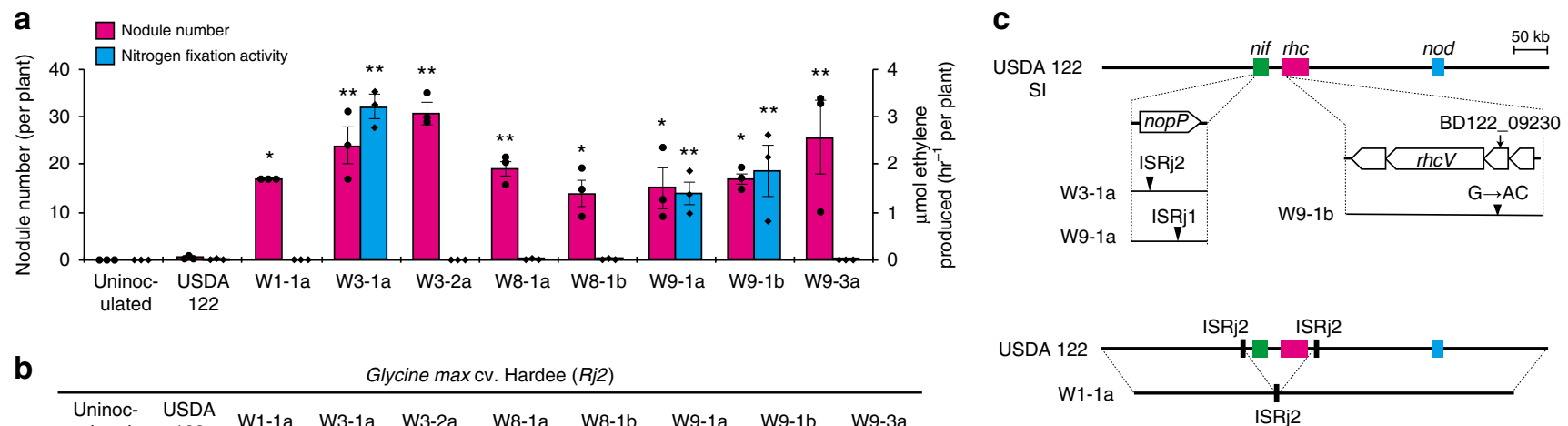

b

Glycine max cv. Hardee (Rj2)
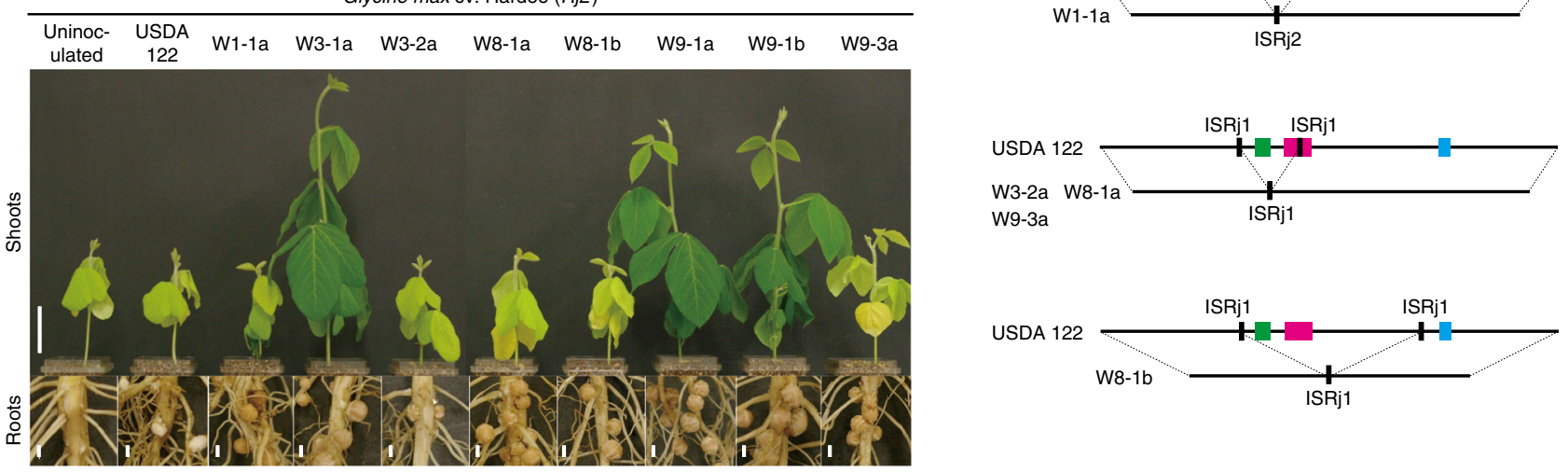

Fig. 2 Characterization of spontaneous mutants overcoming Rj2-mediated incompatibility. a Number of root nodules and nitrogen fixation activity of the roots of G. max cv. Hardee at 28 days after inoculation with USDA 122 and its spontaneous mutants. The data of nodule number shown are representative of two independent experiments. Nitrogen fixation activity was measured by acetylene reduction assay. Error bars show SEM ( $n=3$ ). Significant differences from USDA 122 by Dunnett's multiple comparison test: ${ }^{\star} P<0.05,{ }^{\star \star} P<0.01$. b Shoots and roots of $G$. max cv. Hardee (Rj2) at 28 days after inoculation with USDA 122 or its spontaneous mutants. Scale bars, $5 \mathrm{~cm}$ (for shoots) or $0.5 \mathrm{~cm}$ (for roots). c Positions of mutations in the genomes of spontaneous mutants. Bold horizontal lines indicate the symbiosis island ( $\mathrm{SI}, 672 \mathrm{~kb}$ ). ISRj1 and ISRj2, insertion sequence elements

$-1)$. The average number of nodules was significantly lower on Rj2 transgenic roots inoculated with USDA 122 than on those inoculated with 122 nop $P_{110}$, whereas the average nodule number on roots transformed with $r j 2$ or empty vector was similar after inoculation with USDA 122 and 122 nop $P_{110}$ (Fig. $5 \mathrm{~m}$ ). These results indicate that the residues at positions 452 and 490 of $\mathrm{Rj} 2 /$ rj2 determine the symbiotic compatibility with $B$. diazoefficiens strains having 122-type NopP. Therefore, NopP-mediated Rj2 symbiotic incompatibility is induced via the host $\mathrm{Rj} 2$ protein.

Expressions of defense marker genes in $\mathbf{R j} 2$-soybean roots. To examine whether defense responses are activated under $R j 2$ symbiotic incompatibility, we monitored the expressions of defense marker genes $(P R-1, P R-2$, and $P R-5)$ in Hardee roots. The expression of $P R-2$ was significantly increased at 2 DAI with USDA 122 as compared with 122 nop $P_{110}$ inoculation and uninoculated control (Fig. 6), and markedly decreased at 4 DAI, suggesting the transient and early expression of $P R-2$ elicited by wild-type USDA 122 (Fig. 6). In contrast, the expression of PR-1 and $P R-5$ was not significantly changed (Supplementary Fig. 9). A significant change in Rj2 expression was not observed (Fig. 6) as reported previously ${ }^{23}$. The expression of ENOD40, a wellcharacterized symbiosis gene that is expressed after the NF perception $^{29}$, was induced in Hardee roots at 4 DAI by the inoculation with both USDA 122 and 122 nop $P_{110}$ strains (Fig. 6). These results suggested that, in $R j 2$ symbiotic incompatibility, NF perception and further symbiosis signaling is activated but failed to induce normal nodulation by host defense response.

Amino acid residues of NopP responsible for incompatibility. To determine which of the four amino acid residues in NopP that differed between USDA 122 and $110^{\mathrm{T}}$ (Fig. 4b) are responsible for
Rj2 symbiotic incompatibility, we complemented the nopP deletion mutant of USDA $110^{\mathrm{T}}(110 \Delta n o p P)$ with DNAs encoding each of the 16 possible NopP variants (110nopP_v1 to 110 nopP_v16) that differed in these four residues.

The number of nodules formed on Hardee (Rj2) roots after inoculation with the 16 strains is shown in Fig. 7. Plants inoculated with USDA $110^{\mathrm{T}}$ and $110 \Delta$ nopP formed 23-25 nodules per plant, whereas plants inoculated with 110 nopP_v1, which had USDA 122-type nopP, formed very few nodules, similar to those inoculated with USDA 122 or 110 nop $P_{122}$ (Fig. 4). Plants inoculated with 110 nopP_v1 to 110 nopP_v4, which have R60 and H173, formed significantly fewer nodules than those inoculated with $110 \Delta n o p P$. Slightly fewer nodules were formed upon inoculation with $110 n o p P_{-}$v1 or $110 n o p P_{-} v 2$ than with 110nopP_v3 or 110nopP_v4. Indeed, the second experiments with 10 replicates showed that Hardee inoculated with 110nopP_v2 formed significantly fewer nodules than those inoculated with 110nopP_v4 $(P=0.022$ by two-tailed Student's $t$-test) (Supplementary Fig. 10a). Nodule number was not affected by the amino acid residue at position 271 ( $\mathrm{F}$ or L) or by L60 (110nopP_v9 to 110nopP_v16). Plants inoculated a derivative of USDA 122 carrying 110nopP_v4-type nopP (122nopP_m3) showed apparently intermediate nodule numbers as compared with $122 n o p P_{110}$ and USDA 122 (Supplementary Fig. 10b). Taken together, these results indicate that having R60, R67, and H173 in NopP are required for the suppression of root nodule formation on Rj2soybeans.

Diversity and phylogeny of NopP among soybean bradyrhizobia. To evaluate nopP diversity, we searched the NCBI GenBank database for nopP homologs from B. diazoefficiens and B. japonicum (Supplementary Table 2). In 
a

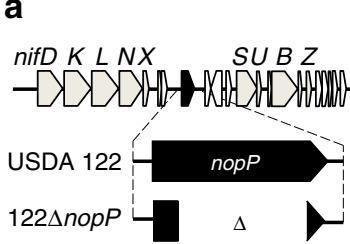

C

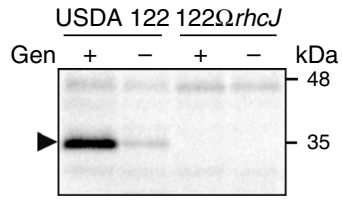

b

nopP (-100 to -63) CTTAGGTCAGGTTGTGGTCAGTTAGGTTGTATATTATG tts-box consensus nnntcGTCAGctTntcGaaAGctnnnccncctAnnnnn

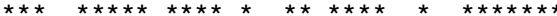

d

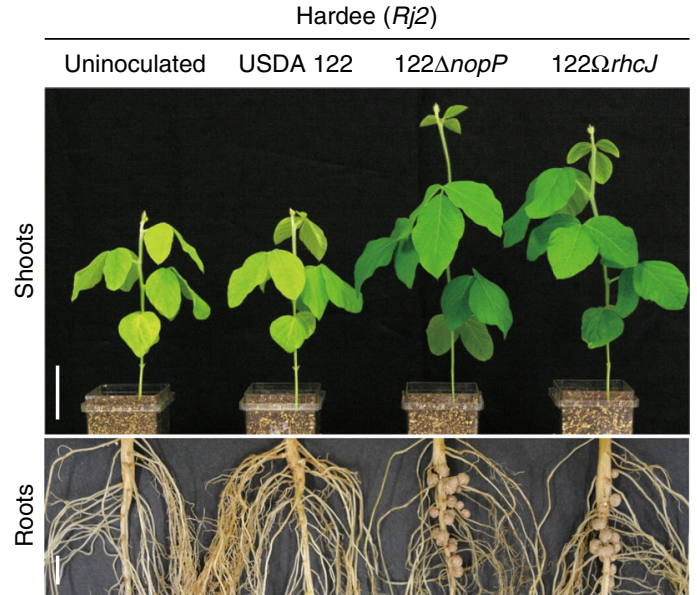

e

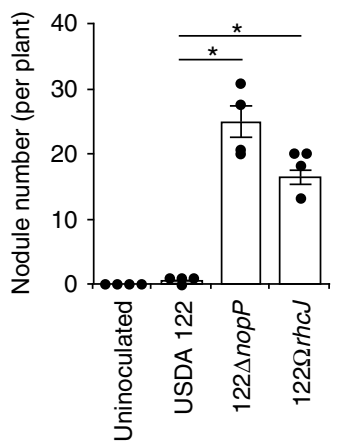

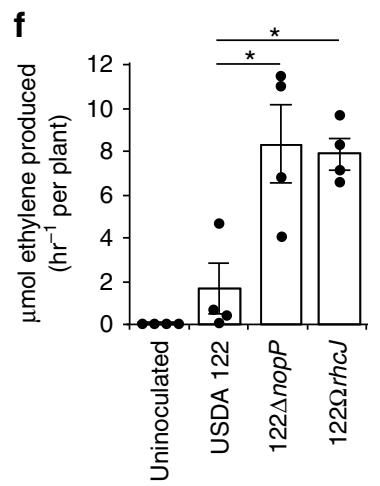

Fig. 3 Characterization of nopP in B. diazoefficiens USDA 122. a

Organization of nopP and its flanking regions in USDA 122 and $122 \Delta$ nopP. $\mathbf{b}$ The tts-box motif found upstream of nopP in USDA 122 and the $t$ ts consensus sequence ${ }^{64}$. Asterisks indicate identical nucleotides. c Western blotting analysis of NopP secreted into culture supernatants from USDA 122 and its mutant with inactivated T3SS $(122 \Omega \mathrm{rhc})$ ). Strains were grown in the presence $(+)$ or absence $(-)$ of $10 \mu \mathrm{M}$ genistein (Gen). The position of NopP is indicated by arrow. d Shoots and roots of G. max cv. Hardee at 28 days after inoculation with USDA 122, 122 $\Delta$ nopP, or $122 \Omega$ rhcJ. Scale bars, $5 \mathrm{~cm}$ (for shoots) or $1 \mathrm{~cm}$ (for roots). e Number of root nodules and $\mathbf{f}$ nitrogen fixation activity. The data of nodule number shown are representative of three independent experiments. Error bars show SEM ( $n$ $=4)$. ${ }^{*}$ Significant difference from USDA $122(P<0.01$ by Dunnett's multiple comparison test)

parallel, we selected phylogenetically diverse $B$. diazoefficiens and B. japonicum strains from our culture collection ${ }^{30}$ (Supplementary Table 2) and sequenced their nopP genes. The deduced amino acid sequences from a total of 55 strains were aligned and classified (Fig. 8a, Supplementary Table 3). The NopP sequence pattern fell into nine types, including NopP of USDA $110^{\mathrm{T}}$ and 122 . The six newly identified types, designated ST1 to ST6, had the same length (277 aa) as the 110 and 122

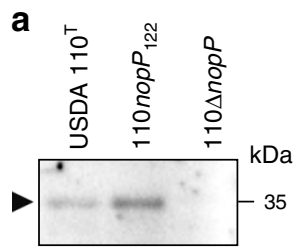

b
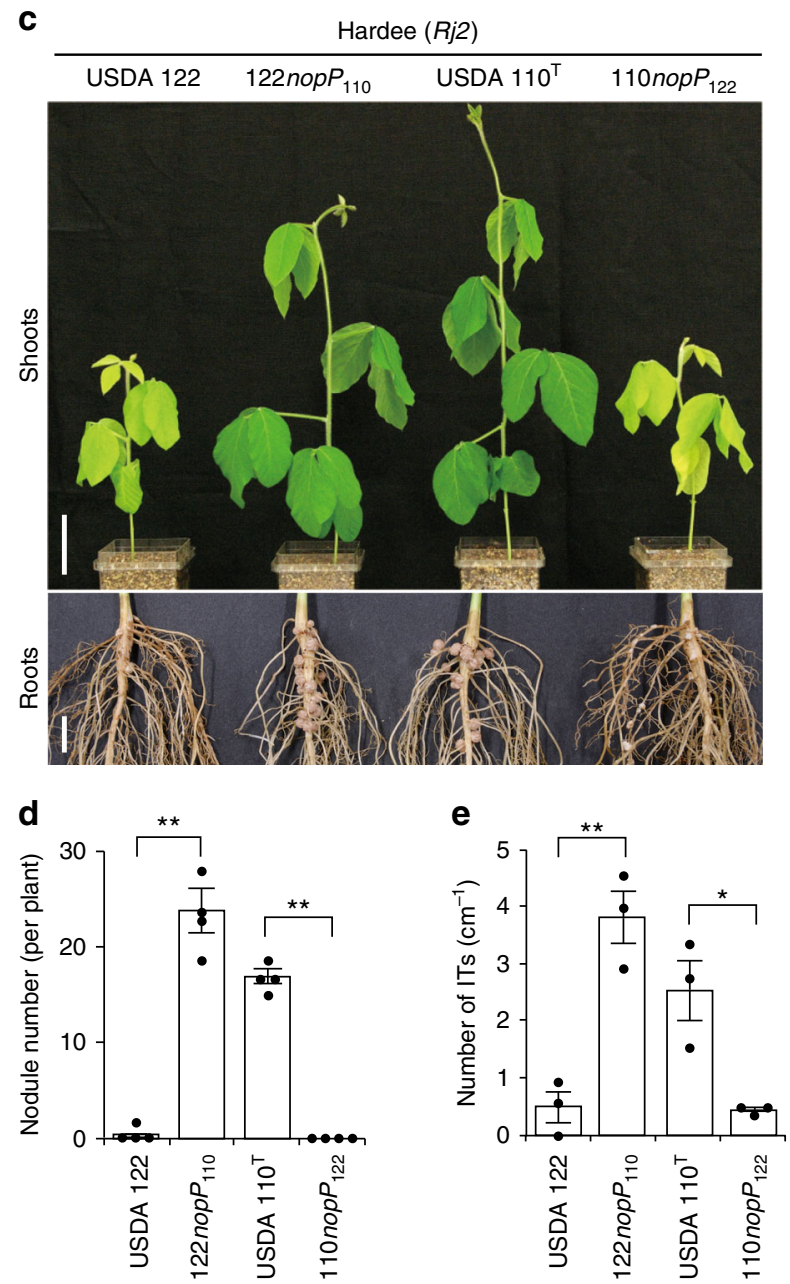

Fig. 4 Effect of nopP swapping between USDA 122 and $110^{\top}$ on symbiosis with Hardee. a Western blotting analysis of NopP secreted into culture supernatants from USDA 110, 110nop $P_{122}$, and nopP deletion mutant of USDA $110^{\top}$ (110 $\triangle$ nopP). Strains were grown in the presence of $10 \mu \mathrm{M}$ genistein. The position of NopP is indicated by arrow. $\mathbf{b}$ Differences in amino acid residues in NopP between B. diazoefficiens USDA 122 and $110^{\top}$. c Shoots and roots of $\mathrm{cv}$. Hardee at 28 days after inoculation with USDA $122,110^{\top}$, or strains with swapped nopP $\left(122\right.$ nop $\left._{110}, 1_{10 \text { nop }} P_{122}\right)$. Scale bars, $5 \mathrm{~cm}$ (for shoots) or $1 \mathrm{~cm}$ (for roots). d Number of nodules formed on roots at 28 days after inoculation. The data shown are representative of three independent experiments. Error bars show SEM $(n=4)$. e Number of infection threads (ITs). ITs were counted on whole region of main roots at 2 days after inoculation. Error bars show $\operatorname{SEM}(n=3)$. ${ }^{\star} P<0.05$, ${ }^{\star \star} P<0.01$ by two-tailed Student's $t$-test

types, and one type was disrupted by the insertion of an IS element (designated "IS-inserted"). Besides positions 60, 67, 173 , and 271, 14 other variations were observed from ST3 to ST6 types (Supplementary Table 3).

In addition to $B$. diazoefficiens strains, some $B$. japonicum strains also have the 122-type gene (Supplementary Table 3). 

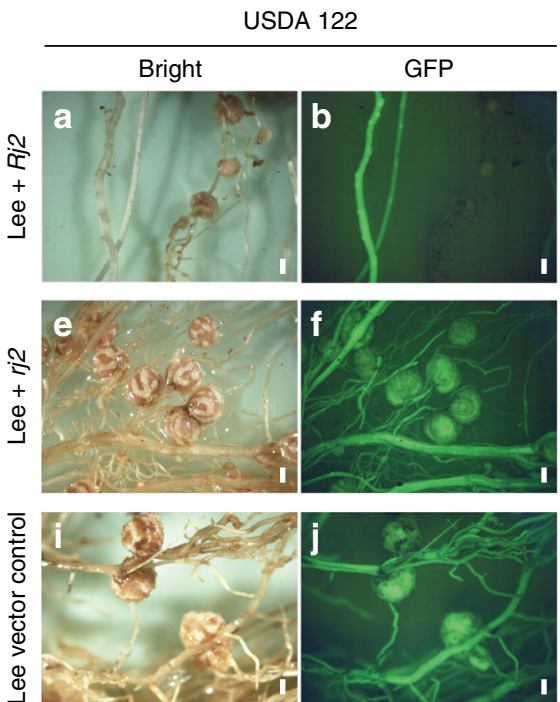
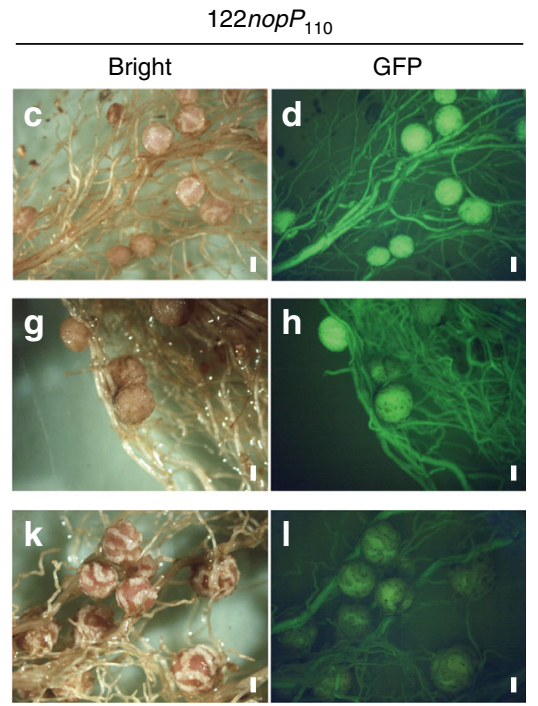

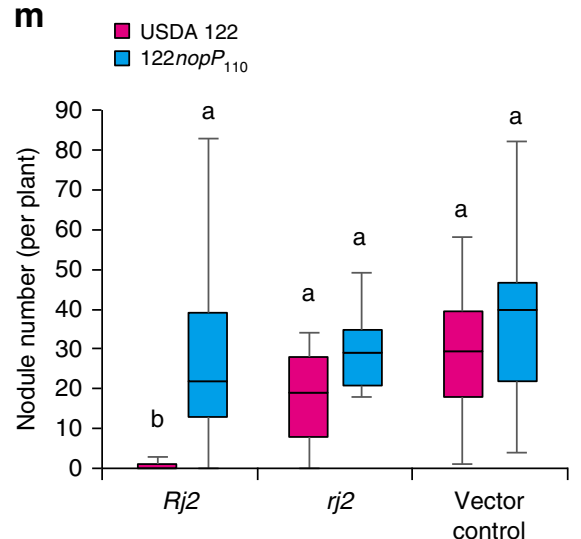

Fig. 5 Nodulation of $r j 2$-soybean transformed with the Rj2 gene by USDA 122 or 122 nop $P_{110}$. $\mathbf{a}, \mathbf{c}, \mathbf{e}, \mathbf{g}, \mathbf{i}, \mathbf{k}$ Bright-field images and $\mathbf{b}$, $\mathbf{d}, \mathbf{f}, \mathbf{h}, \mathbf{j}$, I GFP fluorescence images of roots of transgenic cv. Lee (rj2) complemented with the Rj2, rj2 gene or vector control. B. diazoefficiens USDA 122 or 122 nopP ${ }_{110}$ was inoculated onto each seedling, and images were taken at 4 weeks after inoculation. Scale bars, $1 \mathrm{~mm}$. $\mathbf{m}$ Box-and-whisker plots showing the number of nodules formed on GFP-expressing hairy roots. Combined data from three independent experiments are shown. Center line, median; box limits, first and third quartiles; whiskers, ranges. $n=14$ for $R j 2, n=12$ for $r j 2$, and $n=13$ for vector control. Different letters above bars indicate statistical significance $(P<$ 0.01 by nonparametric Steel-Dwass multiple comparison test)
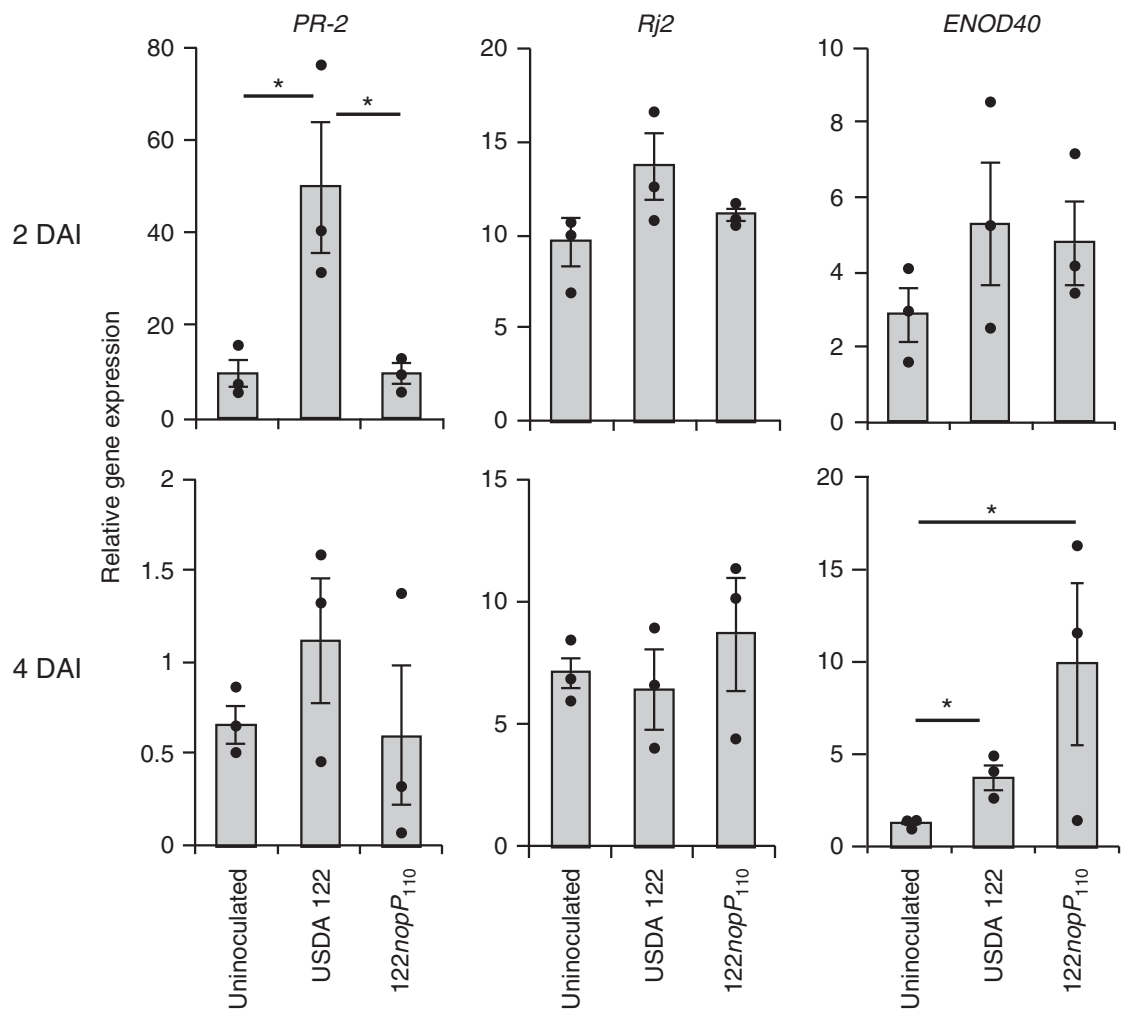

Fig. 6 Expression of $P R-2, R j 2$, and ENOD40 genes in roots of Hardee. Expression of PR-2, Rj2, and ENOD40 in roots of G. max cv. Hardee inoculated with USDA 122 and 122 nopP $P_{110}$ was determined by quantitative reverse-transcription PCR using primers listed in Supplementary Data 2 as described in Methods. RNA was isolated from the roots at 2 and 4 days after inoculation (DAI). The expression level of each gene was normalized by the SUBI2 (ubiquitin) gene. Error bars show SEM of three independent experiments with two plants (from one seed pack). ${ }^{\star} P<0.05$ by two-tailed Student's $t$-test

Such B. japonicum strains (HK7-6, TS4-11, and J5), as well as the ST1-type B. japonicum strain KW1-63 (corresponding to 110nopP_v2 in Fig. 7), did not nodulate on Hardee (Supplementary Fig. 11, Fig. 8a). The NopP sequences of these strains contained R60, R67, and H173 residues, and their symbiotic phenotypes were consistent with the results shown in Fig. 7. Together with the analysis of nopP mutational variants (Fig. 7), these results indicate that a combination of 


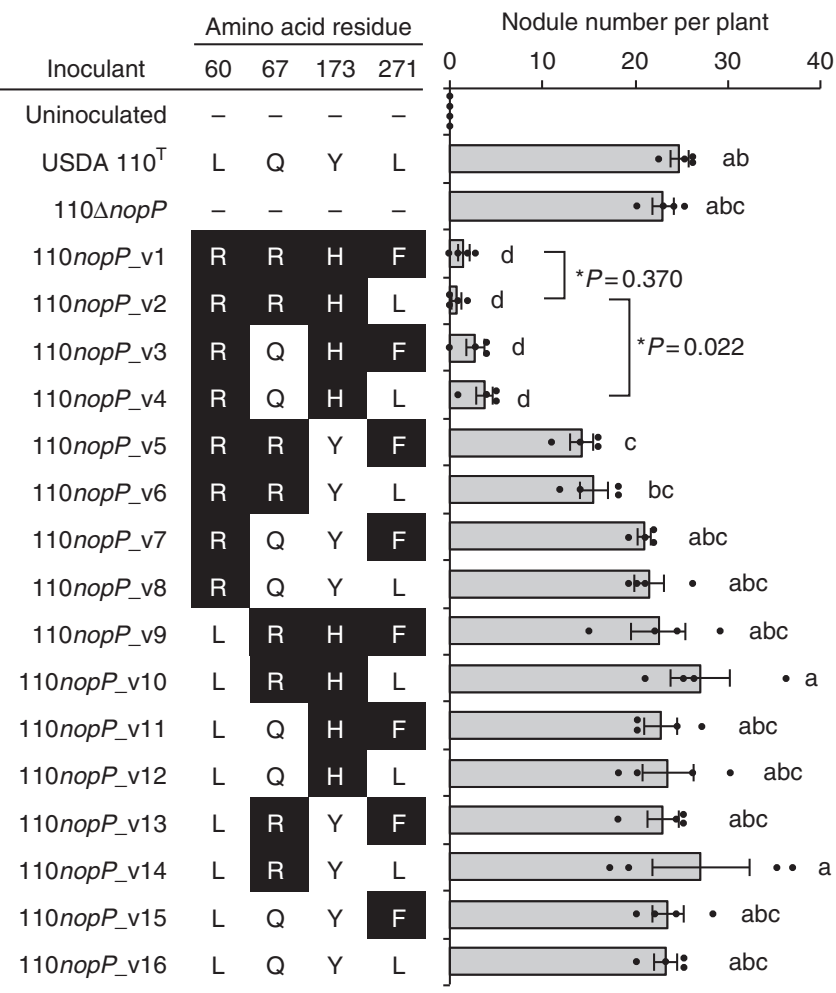

Fig. 7 Nodulation phenotypes of USDA $110^{\top}$ carrying nopP variants on Hardee. Strains and amino acid residues in NopP at positions 60, 67, 173, and 271 are shown on the left, and the nodule numbers at 28 days after inoculation are shown on the right. Error bars show SEM $(n=4)$. Different lowercase letters to the right of the bars indicate significant difference by one-way ANOVA followed by Tukey's honestly significant difference test $(P<0.05)$. The experiment was repeated once again with different comparison as second nodulation assay (Supplementaly Fig. 10a). The asterisks indicate the $P$-values calculated by two-tailed Student's $t$-test based on the data from second nodulation assay $(n=10)$

R60, R67, and H173 is critical for the induction of Rj2 symbiotic incompatibility.

Next, we mapped the NopP types $(122,110$, ST1, and ST2) on a phylogenetic tree of bradyrhizobia based on the 16S-23S rRNA internal transcribed spacer (ITS) sequences (Fig. 8b). USDA 122 and other $B$. diazoefficiens strains possessing 122-type nopP were found in the same cluster within the $B$. diazoefficiens clade, whereas $B$. japonicum strains possessing 122-type nopP were randomly distributed within the B. japonicum clade. Furthermore, the distribution of NopP types (110, 122, ST1, ST2) was not consistent with the bradyrhizobial lineage based on ITS sequences.

Phosphorylation of NopP. NopP from S. fredii NGR234 can be phosphorylated in vitro by plant kinases ${ }^{31}$. In addition, $B$. elkanii may secrete phosphorylated NopP, because several NopP spots with different pI were detected by twodimensional gel electrophoresis of extracellular proteins ${ }^{32}$. To test whether USDA 122 secretes phosphorylated NopP, we analyzed extracellular proteins of USDA 122 in the culture by Phos-Tag SDS-polyacrylamide gel electrophoresis (PAGE) with or without the alkaline phosphatase treatment. The results showed that the alkaline phosphatase had no effect on the mobility of NopP, indicating that it was not phosphorylated (Supplementary Fig. 12). a

\begin{tabular}{ccccccc} 
& \multicolumn{4}{c}{ Amino acid residue } & Nodulation \\
\cline { 2 - 5 } NopP-type & 60 & 67 & 173 & 271 & (cv. Hardee) \\
110 & L & Q & Y & L & + \\
122 & R & R & H & F & - \\
ST1 & R & R & H & L & - \\
ST2 & R & Q & Y & L & + \\
ST3 & R & L & H & I & + \\
ST4 & R & Q & Y & I & + \\
ST5 & R & Q & H & L & + \\
ST6 & R & Q & H & L & + \\
IS-inserted & - & - & - & - & + \\
& & & & & & +
\end{tabular}

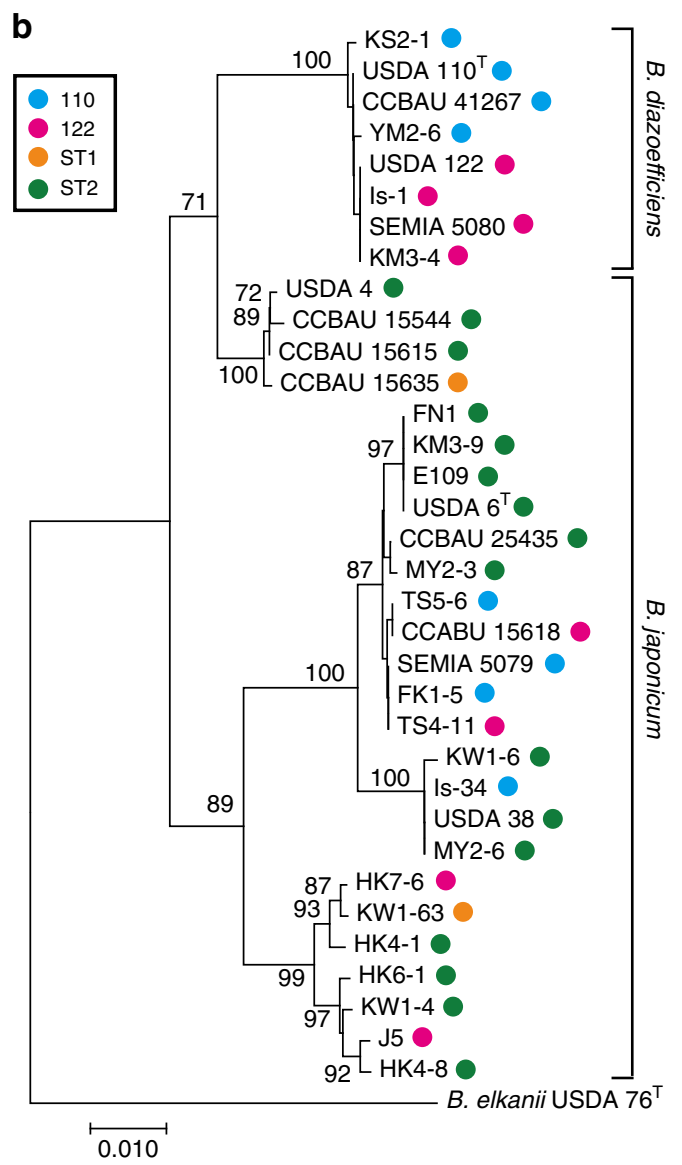

Fig. 8 Diversity of NopP among soybean bradyrhizobia. a Differences in deduced NopP amino acid sequences among $B$. diazoefficiens and $B$. japonicum strains. Amino acid residues in NopP at positions 60, 67, 173, and 271 in each type and nodulation phenotype of a representative strain(s) on G. max cv. Hardee (Supplementary Fig. 11) are shown. b Phylogenetic tree of B. diazoefficiens and B. japonicum based on 16S-23 S rRNA internal transcribed spacer (ITS) sequences. Numbers at the nodes are the percentages of 1000 bootstrap replications supporting that partition (values $\geq 70 \%$ are shown). NopP types (indicated by colored circles) were assigned on the basis of the patterns of amino acid sequences deduced from genomic sequences (Supplementary Table 3). The scale bar shows the number of base substitutions per nucleotide

Spontaneous mutations of nopP in Is-1 mutants. The gene encoding 122-type NopP was found in B. diazoefficiens Is-1 (Supplementary Table 2), which is incompatible with Rj2-soybean plants $^{33}$. Although, Tsurumaru et al. ${ }^{34}$ previously conducted a screening by Tn5-based transposon mutagenesis and obtained eight Rj2-compatible mutants of Is- $1, \operatorname{Tn} 5$ insertions were located in genomic regions other than $r h c$, ttsI, and nopP genes ${ }^{34}$. Thus, 


\begin{tabular}{|c|c|c|c|c|}
\hline Inoculant & $\begin{array}{l}\text { NopP } \\
\text { sequence } \\
\text { type }\end{array}$ & $\begin{array}{l}\text { Nodule } \\
\text { number } a\end{array}$ & $\begin{array}{l}\text { Nodule dry } \\
\text { mass (mg) } \\
a\end{array}$ & $\begin{array}{l}\text { Plant dry } \\
\text { mass } \\
(\mathrm{mg})^{a}\end{array}$ \\
\hline USDA $110^{\top}$ & 110 & $79.7 \pm 4.3$ & $63.6 \pm 3.5$ & $600 \pm 26$ \\
\hline 110 nop $P_{122}$ & 122 & $13.8 \pm 2.4^{\star \star}$ & $35.9 \pm 3.4^{\star \star}$ & $367 \pm 18$ * \\
\hline $110 \Delta$ nopP & Deleted & $61.3 \pm 4.5^{\star \star}$ & $49.6 \pm 5.5$ & $481 \pm 36$ \\
\hline USDA 122 & 122 & $2.4 \pm 0.6$ & $8.5 \pm 2.4$ & $292 \pm 9$ \\
\hline $122 n o p P_{110}$ & 110 & $27.0 \pm 2.6^{\star \star}$ & $34.0 \pm 3.4^{\star \star}$ & $\begin{array}{l}359 \pm \\
25^{\star \star}\end{array}$ \\
\hline $122 \Delta$ nорP & Deleted & $21.7 \pm 1.5^{\star \star}$ & $32.9 \pm 3.6^{\star \star}$ & $348 \pm 26^{\star}$ \\
\hline Uninoculated & - & 0 & 0 & $282 \pm 9$ \\
\hline
\end{tabular}

a Combined data from two independent experiments are shown. Values are mean $\pm \operatorname{SE}(n=11$ for USDA $110^{\top}, n=18$ for USDA 122 , and $n=12$ for the others). Asterisks $\left(^{*}\right)$ indicate significant differences from the wild-type strain (USDA $110^{\top}$ or USDA 122) by Dunnett's multiple

comparison test $\left({ }^{\star} P<0.05,{ }^{\star} P<0.01\right)$

we searched for spontaneous mutations of nopP in the Tn5 mutants ${ }^{34}$. Sanger sequencing revealed mutations caused by an IS element insertion (in the 7C2 mutant) or a nucleotide substitution (in 10C1) within the nopP coding region (Supplementary Table 4). These results suggest that suppression of $R j 2$-mediated incompatibility in at least these two mutants was due to spontaneous mutations of nopP, and that such mutations occur not only in USDA 122, but also in another Rj2-incompatible Bradyrhizobium strain.

Effects of NopP on the symbiosis with mung bean. To investigate whether NopP has a role in the interactions between rhizobia and other legumes, we inoculated wild-type strains of $B$. diazoefficiens and their nopP mutants on Vigna radiata (mung bean) cv. KPS1. Inoculation with the strains having the gene encoding 122-type NopP resulted in significantly fewer nodules and lower plant biomass than inoculation with the strains having the gene encoding 110-type NopP (Table 1). Rj2 orthologs are conserved in the genome of various legumes including $V$. radiata (Supplementary Table 5). Therefore, symbiotic incompatibility mediated by NopP could also occur in leguminous plants other than soybean. In contrast, the plant inoculated with $110 \Delta$ nоp $P$ formed significantly fewer nodules and had a lower biomass than plants inoculated with wild-type USDA $110^{\mathrm{T}}$ (Table 1), suggesting that 110-type NopP, which induced no Rj2mediated incompatibility, is advantageous for nodulation on $V$. radiata cv. KPS1.

\section{Discussion}

Genotype-specific symbiotic incompatibility in legume-rhizobia interactions is an important trait for the use of root nodule bacteria to improve the crop yield ${ }^{35}$. Genetic loci that control root nodule symbiosis of soybean plants have been analyzed for more than 60 years $^{20}$. Thanks to the recent remarkable progress in genomic and genetic technologies, the host plant $R$-genes and rhizobial T3SS $(r h c)$ genes have been found to be responsible for the induction of symbiotic incompatibility $23,24,32$. These findings imply that host plants sense a specific rhizobial T3SS effector via an $\mathrm{R}$ protein, and reject rhizobial infection via a defense response ${ }^{16}$. However, the T3SS effector interacting with the host $\mathrm{R}$ protein, their interaction mechanism, and the signaling pathway that induces genotype-specific incompatibility have remained unknown.

Here we report the rhizobial determinant and propose the molecular mechanism of Rj2 symbiotic incompatibility (Fig. 9).

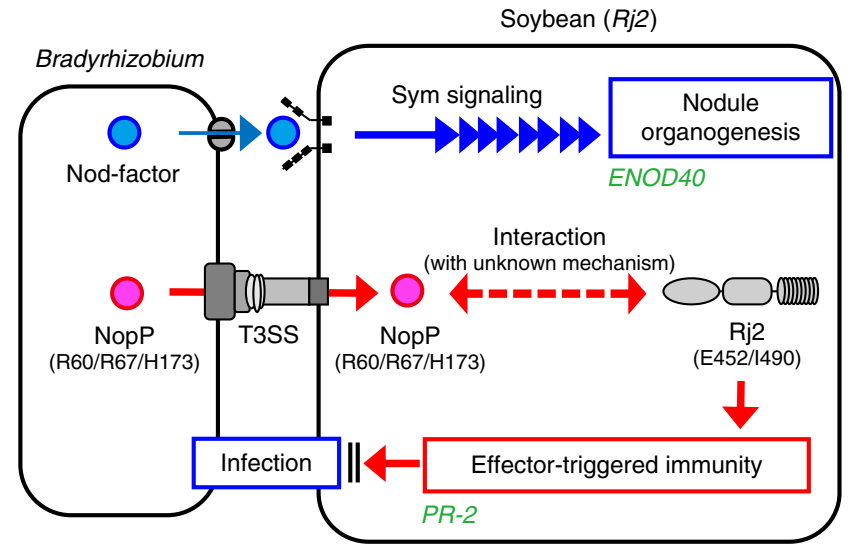

Fig. 9 Proposed molecular mechanism of Rj2 symbiotic incompatibility. Bradyrhizobia produce Nod factors to activate a signaling cascade (symbiosis [Sym] signaling) leading to nodule organogenesis. In the interaction with Rj2-soybeans, incompatible bradyrhizobia inject the NopP effector having the R60, R67, and $\mathrm{H} 173$ residues via T3SS into the host root cell. NopP interacts with the host Rj2 protein with unknown mechanism and activate effector-triggered immunity, which blocks the infection

Using spontaneous mutants of $B$. diazoefficiens USDA 122, we identified a rhizobial T3SS effector, NopP, as a causal factor for symbiotic incompatibility between bradyrhizobia and Rj2-genotype soybean plants (Figs. 2, 3). Our mutational and culture collection analyses of nopP revealed that three amino acid residues (R60, R67, and H173) in NopP are required for Rj2-mediated incompatibility (Figs. 4, 7, 8). Complementation analysis confirmed that $R j 2$-mediated incompatibility is associated with both bradyrhizobial NopP and host $\mathrm{Rj} 2$ protein (Fig. 5). Expression of a host defense marker gene $P R-2$ was significantly increased following inoculation with the incompatible strains, whereas a symbiosis marker gene, ENOD40, was induced regardless of the compatibility of inoculants (Fig. 6). These results indicate that $R j 2$-soybeans activate symbiotic signaling process toward nodule organogenesis in response to incompatible rhizobia, but constantly monitor the specific variants of NopP and reject rhizobial infection by activating Rj2-mediated ETI (Fig. 9).

The first nopP homolog was identified as a host-inducible gene in $S$. fredii USDA $201^{36}$. Subsequent studies revealed that nopP encodes an effector protein secreted by T3SS and inactivation of nopP in $S$. fredii altered nodulation positively or negatively depending on the host plant ${ }^{26,37}$. Schechter et al. ${ }^{38}$ demonstrated that $S$. fredii injects NopP into Vigna unguiculata nodule cells in a T3SS-dependent manner. In response to a flavonoid, $B$. diazoefficiens USDA 122 secretes NopP into culture supernatants through T3SS (Fig. 3), similar to other bradyrhizobia ${ }^{27,32}$. The above data suggest that bradyrhizobial NopP is injected via T3SS into plant cells.

The $\mathrm{Rj} 2$ protein belongs to the TIR-NBS-LRR class of $\mathrm{R}$ proteins. In plant-pathogen interactions, NBS-LRR proteins typically interact with corresponding avirulence (Avr) effector proteins to induce $\mathrm{ETI}^{3}$, which is associated with the hypersensitive response (localized programmed cell death to restrict pathogen growth in plant cells). Most plant resistance proteins belong to the NBSLRR superfamily directly or indirectly recognize pathogen's effectors to activate defense signaling 3,39 . Our results draw parallels between plant-pathogen and plant-mutualist interactions, because NopP acts as an Avr protein that is necessary for USDA 122 to trigger $R j 2$-dependent incompatibility. That incompatibility and compatibility differ by few amino acid residues in NopP protein, which is different from previous observations that epitopes in effector proteins have a more important role ${ }^{39-41}$. 
Rhizobial NopP is not predicted to contain any known functional domains and has no homology with any Avr effectors in pathogens, suggesting that NopP is evolved in symbiotic rhizobial systems. Our future study includes the analysis of direct interactions between those proteins and the screening for NopPinteracting host proteins to understand the recognition of NopP effector by the host $\mathrm{R}$ protein.

In general, ETI involves rapid synthesis of salicylic acid (SA) is rapidly synthesized as an essential signal molecule in response to Avr effector, subsequently inducing SA-dependent defense-signaling marker gene (e.g., $P R-1, P R-2$, and $P R-5)^{42,43}$. In incompatibility response of $R j 4$-soybean plants with $B$. elkanii USDA $61, \mathrm{SA}$ is also accumulated and $P R-1$ expression was increased in roots $^{44}$. Following inoculation with USDA 122, PR-2 gene in Rj2soybeans was transiently induced at an early stage of the infection (2 DAI, Fig. 6). The timing of PR-2 induction was correlated with the inhibition of IT development in root hairs of Hardee (Fig. 4e). Therefore, the results suggest that Rj2-soybeans activate host defense response by inoculation with USDA 122 at an early stage of nodulation and downstream defense-signaling pathway may be different from Rj4-symbiotic incompatibility.

Homologs of nopP are present in rhizobia ${ }^{26}$, but not in pathogens. All known Bradyrhizobium soybean symbionts possess nopP in the middle of the nif gene cluster (Fig. 3a, Supplementary Fig. 7a). These facts imply that NopP plays an important role in symbiotic nitrogen fixation. USDA $110^{\mathrm{T}}$ elicited significantly more nodules on a mung bean cultivar than did $110 \Delta$ nopP (Table 1), but this effect of 110-type nopP was not observed on either Rj2-soybean cv. Hardee (Fig. 7). Thus, USDA 110-type nopP in Bradyrhizobium strains likely has a positive role in nodulation in a host plant-dependent manner, which is consistent with the reports for nopP in $S$. fredii ${ }^{31,37}$. It is still unclear why USDA 122 secretes NopP that is disadvantageous for symbiosis with Rj2-soybeans. A possible explanation is that 122-type NopP may be advantageous for symbiosis with certain other plant genotypes.

Little is known about the importance and evolutionary aspects of genotype-specific symbiotic incompatibility between legumes and rhizobia. In plant-pathogen interactions, compatibility has been proposed to be the consequence of an evolutionary arms race ${ }^{45,46}$. As symbiotic compatibility changes as a result of substitutions of only a few residues in the host $\mathrm{R}$ protein $^{23}$ and rhizobial NopP (Fig. 9), Rj2-mediated incompatibility may be part of the evolutionary arms race to facilitate coevolution between both partners. The mutants of USDA 122 (W3-1a, W9-1a) and Is-1 (7C2, 10C1) carried a mutated nopP and avoided the selection pressure from $R j 2$-soybeans (Fig. 2, Supplementary Table 4). Variation in this gene seems to have occurred independently in different bradyrhizobial lineages (Fig. 8). Thus, we suggest that bradyrhizobia often evolve nopP to circumvent the plant immune system. Furthermore, NopPmediated incompatibility observed in a mung bean cultivar (Table 1) and Rj2 orthologs are conserved in various legume species (Supplementary Table 5). These evidences suggest that determination of compatibility via NopP and R proteins is widely distributed in legumes with nodule symbiosis.

\footnotetext{
Methods

Bacterial strains and growth conditions. Bacterial strains are listed in Supplementary Data 1 . Bradyrhizobium strains were grown aerobically at $30{ }^{\circ} \mathrm{C}$ in HEPES-MES (HM) salt medium ${ }^{47}$ (supplemented with $0.1 \%$ arabinose and $0.025 \%$ [wt/vol] yeast extract). Escherichia coli strains were grown at $37^{\circ} \mathrm{C}$ in Luria-Bertani medium ${ }^{48}$. Agrobacterium rhizogenes was grown at $28^{\circ} \mathrm{C}$ in Yeast Extract Peptone medium ${ }^{49}$. The following antibiotics were added: for Bradyrhizobium strains, kanamycin $(\mathrm{Km})$ at $100 \mathrm{mg} \mathrm{l}^{-1}$, spectinomycin (Sp) at $100 \mathrm{mg} \mathrm{l}^{-1}$, and streptomycin $(\mathrm{Sm})$ at $100 \mathrm{mgl}^{-1}$, or polymyxin $\mathrm{B}(\mathrm{Px})$ at $50 \mathrm{mgl}^{-1}$; for E. coli, ampicillin
}

(Ap) at $50 \mathrm{mg} \mathrm{l}^{-1}, \mathrm{Sp}$ at $25 \mathrm{mg} \mathrm{l}^{-1}$, and $\mathrm{Sm}$ at $25 \mathrm{mg} \mathrm{l}^{-1}$, or $\mathrm{Km}$ at $50 \mathrm{mg} \mathrm{l}^{-1}$; for $A$. rhizogenes, chloramphenicol at $30 \mathrm{mg} \mathrm{l}^{-1}$ or $\mathrm{Km}$ at $100 \mathrm{mgl}^{-1}$.

DNA manipulations. The isolation of plasmid DNA, restriction enzyme digestions, DNA ligations, and the transformation of $E$. coli were done as described by Sambrook and Russell ${ }^{50}$. Plasmids used in this study are listed in Supplementary Data 1. Oligonucleotide primers for PCR are listed in Supplementary Data 2.

Plant growth conditions and nodulation assays. The soybean cultivars used in the study were G. $\max (\mathrm{L}$.) Merr. cv. Hardee (Rj2), CNS (Rj2), IAC-2 (Rj2), and Lee (rj2). The mung bean cultivar was $V$. radiata cv. KPS1. For nodule counting and acetylene-reducing activity (ARA) assays, seeds of $G$. max and $V$. radiata were surface sterilized in $0.5 \%$ sodium hypochlorite for $1 \mathrm{~min}$ and then washed 10 times with sterile distilled water. The seeds were sown in a $300 \mathrm{ml}$ plant box (CUL JAR300; Iwaki, Tokyo, Japan) containing sterile vermiculite and watered with a nitrogen-free plant nutrient solution ${ }^{51}$. Bradyrhizobium strains were cultured for 5 or 6 days. The number of cells was adjusted to $10^{7} \mathrm{ml}^{-1}$ in sterile water by direct counting with a Thoma hemocytometer (Kayagaki Irika Kogyo Co. Ltd., Tokyo, Japan), and $1 \mathrm{ml}$ aliquots were inoculated onto surface-sterilized seeds. Plants were grown in a plant growth cabinet (NK Systems Co. Ltd., Osaka, Japan) at $25^{\circ} \mathrm{C}$ with a photoperiod of $16 \mathrm{~h}$ light $/ 8 \mathrm{~h}$ dark. The number of nodules and the dry weights of nodule and plant were determined 28 DAI. The ARA of roots was analyzed as described by Itakura et al. ${ }^{52}$ Briefly, the roots were transferred into a $100 \mathrm{ml}$ vials (SVG-100, Nichidenrika-glass, Co. Ltd., Kobe, Japan) and acetylene gas was injected at a final concentration of $10 \%(\mathrm{vol} / \mathrm{vol})$. The vial was incubated for $30 \mathrm{~min}$ at $25^{\circ} \mathrm{C}$. The ethylene concentration was determined with a Shimadzu GC-18A gas chromatograph (Shimadzu Co., Kyoto, Japan) equipped with a flame ionization detector and a Porapack N column (GL Sciences, Inc., Tokyo, Japan).

For observation of early infection event and evaluation of gene expression in soybean roots, surface-sterilized Hardee seeds were germinated at $28^{\circ} \mathrm{C}$ for 3 days on two-layered moistened kimwipes. Two seedlings were aseptically transferred to a seed pack (Daiki Rika Co. Ltd., Saitama, Japan) with nitrogen-free medium. One day after transplantation, the seedlings were inoculated with $1 \times 10^{7}$ cells (in 0.1 $\mathrm{ml}$ ) of $B$. diazoefficiens strains or $0.1 \mathrm{ml}$ sterilized water (uninoculated control). Plants were cultivated in a growth chamber at $25^{\circ} \mathrm{C}$ and $70 \%$ humidity with a photoperiod of $16 \mathrm{~h}$ light $/ 8 \mathrm{~h}$ dark. For observation of infection threads formed in root hair inoculated with a gusA-tagged strain, 2-day root samples were immersed in a $\beta$-glucuronidase staining solution [ $100 \mathrm{mM}$ phosphate buffer $(\mathrm{pH} 7.0), 0.5 \mu \mathrm{M}$ $\mathrm{K}_{3}\left(\mathrm{Fe}(\mathrm{CN})_{6}\right), 0.5 \mu \mathrm{M} \mathrm{K}_{4}\left(\mathrm{Fe}(\mathrm{CN})_{6}\right), 0.3 \%$ Triton $\mathrm{X}-100$, and $200 \mathrm{mg} \mathrm{ml}^{-1}$ of 5 bromo-4-chloro-3-indolyl- $\beta$-d-glucuronic acid]. The roots were subjected to vacuum treatment for $15 \mathrm{~min}$ and incubated overnight at $28^{\circ} \mathrm{C}$. The number of infection threads was counted with an Olympus BX51N Microscope (Olympus, Tokyo, Japan).

Isolation of spontaneous mutants of USDA 122. The wild-type B. diazoefficiens USDA 122 strain was inoculated onto surface-sterilized G. max cv. Hardee seeds and plants were cultivated as described above. After 21 days, nodules were picked from each root, dipped in $70 \%$ ethanol for $5 \mathrm{~s}$, and surface sterilized in $1 \%(\mathrm{w} / \mathrm{v})$ sodium hypochlorite for $5 \mathrm{~min}$. Nodules were washed several times with sterile water and transferred into a $1.5 \mathrm{ml}$ tubes (one nodule per tube) containing $100 \mu \mathrm{l}$ of HM liquid medium. Each nodule was homogenized with a sterile toothpick and the suspension was streaked onto HM agar plates. After 7 days of incubation, single colonies that appeared from each nodule were transferred onto a new HM plate for purification.

Whole-genome resequencing. Total DNA was extracted from a stationary-phase culture of Bradyrhizobium cells by using an illustra bacteria genomicPrep Mini Spin Kit (GE Healthcare UK Ltd., Buckinghamshire, UK) and was processed using a Nextera DNA Sample Preparation Kit (Illumina, San Diego, CA, USA) to generate a shotgun library with unique index adapters. The library from each Bradyrhizobium strain was sequenced on a MiSeq system (Illumina), yielding 250 bp paired-end reads. The raw reads from each Bradyrhizobium genome were trimmed and assembled de novo in CLC Genomic Workbench software (CLC bio, Inc., Aarhus, Denmark). The mutations in spontaneous mutants were identified by comparing the obtained MiSeq short reads and the reference B. diazoefficiens USDA 122 genome sequence (DDBJ/EMBL/GenBank accession number CP013127) using the "missing $k$-mer" tool of ShortReadManager ${ }^{53,54}$.

Western blotting and Phos-tag SDS-PAGE. Rabbit polyclonal antibody to NopP protein (anti-NopP) was raised and affinity-purified against the synthetic peptide CIKGETFREKFGRND (GenScript Japan, Inc.,Tokyo, Japan). Extracellular proteins from the culture supernatants of Bradyrhizobium strains were isolated by phenol extraction $^{27}$ and separated ( $3 \mu \mathrm{g}$ protein per well) by $12.5 \%$ SDS-PAGE. The separated proteins were transferred to PVDF membrane using a semi-dry transfer system (HorizeBLOT 4M-R, ATTO, Tokyo, Japan). The membranes were incubated with anti-NopP (diluted 1:10,000) for $30 \mathrm{~min}$ at room temperature. The membranes were then incubated with horseradish peroxidase-conjugated antirabbit IgG (Promega, Inc., Madison, WI, USA) diluted 1:2500 for $30 \mathrm{~min}$, and the immunoreactive bands were detected using the chemiluminescent substrate, 
Chemi-Lumi One Super (Nacalai Tesque, Inc., Kyoto, Japan). We confirmed that NopP (predicted molecular mass: $31 \mathrm{kDa}$ ) of USDA 122 was detected around $35 \mathrm{kDa}$ (Supplementary Fig. 13). Phosphorylation of NopP protein was investigated using Phos-Tag SDS-PAGE technology, which was developed for mobility shift detection of phosphorylated protein ${ }^{55}$. The polyacrylamide gels consisted of a BisTris acrylamide gel copolymerized with Phos-tag [8\% acrylamide, $20 \mu \mathrm{M}$ acrylamide-pendant Phos-tag (Wako, Inc. Osaka, Japan), $40 \mu \mathrm{M} \mathrm{ZnCl} 2$ ]. After electrophoresis, Phos-Tag gels were subjected to western blotting analysis as described above. Dephosphorization of extracellular proteins was performed according to modified procedures described by Kinoshita et al. ${ }^{55}$. Alkaline phosphatase (CIAP, Takara Bio, Inc., Kusatsu, Japan) was added to extracellular proteins $\left(1 \mathrm{U} \mathrm{\mu g}^{-1}\right.$ protein) in appropriate buffer and incubated at $37^{\circ} \mathrm{C}$ for $0,30,60$, and $120 \mathrm{~min}$. The dephosphorylation reactions were stopped by addition of a halfvolume of $3 \times$ SDS-PAGE loading buffer.

Construction of B. diazoefficiens nopP mutants. A mobilizable plasmid for generating in-frame nopP deletion mutants $(122 \Delta n o p P$ or $110 \Delta n o p P)$ was constructed as follows: a $0.6 \mathrm{~kb}$ fragment containing the $5^{\prime}$-portion of the nopP coding region $(+1$ to $+156 \mathrm{bp})$ and the upstream flanking region and a $0.7 \mathrm{~kb}$ fragment encoding the C-terminal region of nopP $(+823$ to $+834 \mathrm{bp})$ and containing the 3 -flanking region were amplified by PCR. The two PCR products were fused by overlap extension PCR and the fused fragment was cloned into the SmaI site of pK18mobsacB ${ }^{56}$. The resulting plasmid (pMS130) was transferred from E. coli DH5a into B. diazoefficiens USDA 122 and $110^{\mathrm{T}}$ by triparental mating with the mobilizing strain E. coli HB101 harboring the pRK2013 helper plasmid ${ }^{57}$. A Km/Px-resistant and sucrose-sensitive transconjugant was selected for single-crossover insertion of the plasmid into the chromosome. The cells were grown in HM liquid medium and were spread on HM agar medium containing Px and 10\% (w/v) sucrose to rescreen for sucrose-resistant colonies. The selected clones were further screened for Km sensitivity. Double-crossover events and an in-frame deletion of nopP were confirmed by PCR and Sanger sequencing in a 3730xl DNA Analyzer with a BigDye Terminator Cycle Sequencing Reaction Kit (Thermo Fisher Scientific, Inc., Waltham, MA, USA).

To swap nopP between USDA 122 and $110^{\mathrm{T}}$, the fragments containing $1.9 \mathrm{~kb}$ $n o p P$ and its flanking region were amplified by PCR from genomic DNA and cloned into the SmaI site of pK18mobsacB. The resulting plasmid pMS127 (containing nopP of USDA 122 [nop $P_{122}$ ]) or pMS128 (containing nopP of USDA $110^{\mathrm{T}}\left[\right.$ nop $\left.\left.P_{110}\right]\right)$ was transformed into USDA $110^{\mathrm{T}}$ or 122 , respectively. The candidate double-crossover mutants $\left(122 n o p P_{110}\right.$ or $\left.110 n o p P_{122}\right)$ were selected as described above. nopP sequences were confirmed by Sanger sequencing. $122 n o p P \_m 3$ was obtained as a by-product of constructing strain $122 n o p P_{110}$.

Construction of mutated nopP variants. Sixteen $1.5 \mathrm{~kb}$ fragments containing the $n o p P$ coding and flanking regions and carrying the target nucleotide substitutions were obtained by overlap extension PCR with unique oligonucleotide primers (Supplementary Data 2). Each of these fragments was inserted into the SmaI site of pK18mob ${ }^{56}$, yielding pMS155 to pMS170 (Supplementary Data 1). These plasmids were transferred into the nopP deletion mutant of USDA $110^{\mathrm{T}}(110 \Delta n o p P)$ by triparental mating as described above. A $\mathrm{Km} / \mathrm{Px}$-resistant transconjugant was selected for single-crossover insertion of each plasmid into the chromosome, yielding $110 \Delta n o p P_{\mathrm{v} 1}$ to $110 \Delta n o p P_{\mathrm{v} 16}$. The single-crossover events were confirmed by PCR, and $n o p P$ sequences were verified by Sanger sequencing.

Rj2 complementation test using hairy root transformation. To obtain a cDNA fragment corresponding to the Rj2 gene, total RNA was extracted from G. max $\mathrm{cv}$. Hardee nodules formed by inoculation with $B$. diazoefficiens USDA $110^{\mathrm{T}}$ with a NucleoSpin RNA Plant Kit (Macherey-Nagel, Inc., Düren, Germany) according to the manufacturer's instructions. First-strand cDNA was synthesized using a SuperScript III First Strand cDNA Synthesis System (Thermo Fisher Scientific, Inc.) and the specific oligonucleotide primer Rj2-3UTR_R3. The first-strand cDNA was subjected to PCR with Rj2_Y2H_F2 and Rj2_Y2H_R3 primers to amplify the full-length $R j 2 \mathrm{cDNA}$ fragment, and the product was subsequently cloned into the pENTR-dTOPO vector (Thermo Fisher Scientific, Inc.), yielding pMS145 as an entry clone for the Gateway Cloning System. To generate rj2 cDNA, G1354 and T1469 (corresponding to E452 and I490 in the protein) in Rj2 cDNA were substituted with A1354 and G1469 by site-directed mutagenesis using overlap extension $\mathrm{PCR}^{58}$. The DNA fragment obtained was cloned into the same vector, yielding pMS154 as an entry clone. cDNAs were transferred from the entry clones into the binary vector pUB-GW-GFP 28 between the polyubiquitin ( $L j U b q 1)$ promoter and the nos terminator using Gateway LR Clonase II (Thermo Fisher Scientific, Inc.). These constructs were used to transfect $A$. rhizogenes K599. Six-day-old seedlings of rj2-soybean cv. Lee were used for complementation testing; A. rhizogenesmediated hairy root transformation was based on the protocol described by Kereszt et al. ${ }^{59}$ In brief, the cotyledonary node was infected with overnight cultures of K599 carrying Rj2 cDNAs (E452/I490 or K452/R490) using a syringe. Infected seedlings were maintained in sterile vermiculite pots in a growth chamber under high humidity until hairy roots developed at the infection site $(\sim 2$ weeks). The seedlings, with the main roots and non-GFP roots removed under fluorescence binocular microscope, were cultured in sterile vermiculite pots containing $1 / 2 \mathrm{~B} \& \mathrm{D}$ solution ${ }^{60}$ with $0.5 \mathrm{mM} \mathrm{NH}_{4} \mathrm{NO}_{3}$ for 1 week. The seedlings were inoculated with $B$. diazoefficiens USDA 122 (wild type or 122 nop $P_{110}$ ) under nitrogen-free conditions ${ }^{51}$ and nodulation on transgenic roots was examined 4 weeks after inoculation.

Quantitative RT-PCR of genes in the roots of soybean. Two plants were sampled from a seed pack at 2 or $4 \mathrm{DAI}$ and the main roots were merged in a $15 \mathrm{ml}$ tube. The roots were immediately frozen in liquid nitrogen and kept at $-80^{\circ} \mathrm{C}$ until RNA extraction. Total RNA was extracted using a NucleoSpin RNA Plant Kit (Macherey-Nagel, Inc.) and additionally treated with DNase I (Promega, Inc.) according to the manufacturer's instructions. First-strand cDNA was synthesized using a SuperScript III First Strand cDNA Synthesis System for reversetranscription PCR (RT-PCR) (Thermo Fisher Scientific, Inc.) with random hexamers. Relative expression was analyzed by quantitative RT-PCR in a LightCycler Nano Instrument (Roche, Basel, Switzerland) using FastStart Essential DNA Green Master (Roche) and specific primers (Supplementary Data 2). The PCR reaction mixture was adjusted according to the manufacturer's instructions and thermal cycling conditions consisted of $10 \mathrm{~min}$ at $95^{\circ} \mathrm{C}$, and 45 cycles of $20 \mathrm{~s}$ at $95^{\circ} \mathrm{C}, 20 \mathrm{~s}$ at $55^{\circ} \mathrm{C}$, and $20 \mathrm{~s}$ at $72^{\circ} \mathrm{C}$. The specificity of PCR amplification was confirmed by a melting-curve analysis. Transcript levels were normalized to the expression of SUBI2 (ubiquitin) measured in the same samples ${ }^{61}$.

nopP diversity and phylogenetic analysis. Total DNA was extracted from the cells of Bradyrhizobium strains in the Japanese culture collection ${ }^{30}$ by using an illustra bacteria genomicPrep Mini Spin Kit (GE Healthcare UK, Ltd.). nopP with its flanking regions was amplified by PCR with the oligonucleotide primers nopP_F1 and nopP_R1. Purified PCR products were subjected to Sanger sequencing with the primers nopP_F1, nopP_R1, nopP_F2, and nopP_R3. The obtained sequences were assembled and amino acid sequences were deduced in GenetyxMAC v. 18.0.3 software (Genetyx Co., Tokyo, Japan). The ITS sequences between the $16 \mathrm{~S}$ and $23 \mathrm{~S}$ rRNA genes for phylogenetic analysis are listed in Supplementary Table 2. The sequences were aligned using the CLUSTALW program ${ }^{62}$. Neighborjoining trees were constructed in MEGA v. 7 software $^{63}$ and 1000 bootstrap replicates were used to generate a consensus tree.

Statistical analysis. Statistical significance was determined using a two-tailed Student's $t$-test performed by Microsoft Excel for pairwise comparisons. A one-way analysis of variance followed by a Dunnett's test or by Tukey's honest significant difference test was performed using R 3.3.2 software (https://www.r-project.org/) with "multcomp" package for comparisons of multiple test samples. For nonnormal distribution data, significance of difference among groups was evaluated by Steel-Dwass test using JMP software (SAS Institute Inc. Cary, NC, USA). $P$-values $<0.05$ were considered statistically significant. Sample size $(n)$ used for experiments is indicated in the figure legends.

Data availability. MiSeq sequences were deposited in the DDBJ Sequence Read Archive DRA005835 [DRX086799 to DRX086806] and DRA005773 [DRX085569]. DNA sequences of the nopP genes determined by the Sanger method were deposited in the GenBank/EMBL/DDBJ under the accession codes (LC331619 to LC331646, and LC331679 to LC331683) listed in Supplementary Table 2. All relevant data that support the findings of this study are available from the corresponding author upon reasonable request.

Received: 1 December 2017 Accepted: 12 July 2018 Published online: 07 August 2018

\section{References}

1. Galán, J. E. \& Collmer, A. Type III secretion machines: bacterial devices for protein delivery into host cells. Science 284, 1322-1328 (1999).

2. He, S. Y. Type III protein secretion systems in plant and animal pathogenic bacteria. Annu. Rev. Phytopathol. 36, 363-392 (1998).

3. Gassmann, W. \& Bhattacharjee, S. Effector-triggered immunity signaling: from gene-for-gene pathways to protein-protein interaction networks. Mol. Plant Microbe Interact. 25, 862-868 (2012).

4. Bohlool, B. B., Ladha, J. K., Garrity, D. P. \& George, T. Biological nitrogen fixation for sustainable agriculture: a perspective. Plant Soil 141, 1-11 (1992)

5. Wagner, S. C. Biological nitrogen fixation. Nat. Educ. Knowl. 3, 15 (2011).

6. Dénarié, J. \& Cullimore, J. Lipo-oligosaccharide nodulation factors-a new class of signaling molecules mediating recognition and morphogenesis. Cell 74, 951-954 (1993).

7. Radutoiu, S. et al. LysM domains mediate lipochitin-oligosaccharide recognition and Nfr genes extend the symbiotic host range. EMBO J. 26, 3923-3935 (2007). 
8. Spaink, H. P. The molecular basis of infection and nodulation by rhizobia: the ins and outs of sympathogenesis. Annu. Rev. Phytopathol. 33, 345-368 (1995).

9. Deakin, W. J. \& Broughton, W. J. Symbiotic use of pathogenic strategies: rhizobial protein secretion systems. Nat. Rev. Microbiol. 7, 312-320 (2009).

10. Miwa, H. \& Okazaki, S. How effectors promote beneficial interactions. Curr. Opin. Plant. Biol. 38, 148-154 (2017).

11. Okazaki, S., Kaneko, T., Sato, S. \& Saeki, K. Hijacking of leguminous nodulation signaling by the rhizobial type III secretion system. Proc. Natl Acad. Sci. USA 110, 17131-17136 (2013)

12. Tampakaki, A. P. Commonalities and differences of T3SSs in rhizobia and plant pathogenic bacteria. Front. Plant Sci. 5, 114 (2014).

13. Marie, C., Broughton, W. J. \& Deakin, W. J. Rhizobium type III secretion systems: legume charmers or alarmers? Curr. Opin. Plant. Biol. 4, 336-342 (2001).

14. Krause, A., Doerfel, A. \& Göttfert, M. Mutational and transcriptional analysis of the type III secretion system of Bradyrhizobium japonicum. Mol. Plant Microbe Interact. 15, 1228-1235 (2002).

15. Wassem, R. et al. TtsI regulates symbiotic genes in Rhizobium species NGR234 by binding to tts boxes. Mol. Microbiol. 68, 736-748 (2008).

16. Gourion, B., Berrabah, F., Ratet, P. \& Stacey, G. Rhizobium-legume symbioses: the crucial role of plant immunity. Trends Plant. Sci. 20, 186-194 (2015)

17. Kimbrel, J. A. et al. Mutualistic co-evolution of type III effector genes in Sinorhizobium fredii and Bradyrhizobium japonicum. PLoS Pathog. 9, e1003204 (2013).

18. Delamuta, J. R. et al. Polyphasic evidence supporting the reclassification of Bradyrhizobium japonicum group Ia strains as Bradyrhizobium diazoefficiens sp. nov. Int. J. Syst. Evol. Microbiol. 63, 3342-3351 (2013).

19. van Berkum, P. \& Fuhrmann, J. J. Evolutionary relationships among the soybean bradyrhizobia reconstructed from 16S rRNA gene and internally transcribed spacer region sequence divergence. Int. J. Syst. Evol. Microbiol. 50, 2165-2172 (2000).

20. Hayashi, M. et al. $R j(r j)$ genes involved in nitrogen-fixing root nodule formation in soybean. Breed. Sci. 61, 544-553 (2012)

21. Caldwell, B. E. Inheritance of a strain-specific ineffective nodulation in soybeans. Crop Sci. 6, 427-428 (1966).

22. Caldwell, B. E. \& Vest, G. Nodulation interactions between soybean genotypes and serogroups of Rhizobium japonicum. Crop Sci. 8, 680-682 (1968).

23. Yang, S., Tang, F., Gao, M., Krishnan, H. B. \& Zhu, H. R gene-controlled host specificity in the legume-rhizobia symbiosis. Proc. Natl Acad. Sci. USA 107, 18735-18740 (2010).

24. Tsukui, T. et al. The type III secretion system of Bradyrhizobium japonicum USDA122 mediates symbiotic incompatibility with Rj2 soybean plants. Appl. Environ. Microbiol. 79, 1048-1051 (2013).

25. Sugawara, M. et al. Complete genome sequence of Bradyrhizobium diazoefficiens USDA 122, a nitrogen-fixing soybean symbiont. Genome Announc. 5, e01743-16 (2017).

26. Ausmees, N. et al. Characterization of NopP, a type III secreted effector of Rhizobium sp. strain NGR234. J. Bacteriol. 186, 4774-4780 (2004).

27. Süß, C. et al. Identification of genistein-inducible and type III-secreted proteins of Bradyrhizobium japonicum. J. Biotechnol. 126, 69-77 (2006).

28. Maekawa, T. et al. Polyubiquitin promoter-based binary vectors for overexpression and gene silencing in Lotus japonicus. Mol. Plant Microbe Interact. 21, 375-382 (2008).

29. Kouchi, H. \& Hata, S. Isolation and characterization of novel nodulin cDNAs representing genes expressed at early stages of soybean nodule development. Mol. Gen. Genet. 238, 106-119 (1993).

30. Shiina, Y. et al. Relationship between soil type and $\mathrm{N}(2) \mathrm{O}$ reductase genotype (nos $Z$ ) of indigenous soybean bradyrhizobia: nos Z-minus populations are dominant in Andosols. Microbes Environ. 29, 420-426 (2014).

31. Skorpil, P. et al. NopP, a phosphorylated effector of Rhizobium sp. strain NGR234, is a major determinant of nodulation of the tropical legumes Flemingia congesta and Tephrosia vogelii. Mol. Microbiol. 57, 1304-1317 (2005).

32. Okazaki, S., Zehner, S., Hempel, J., Lang, K. \& Göttfert, M. Genetic organization and functional analysis of the type III secretion system of Bradyrhizobium elkanii. FEMS Microbiol. Lett. 295, 88-95 (2009)

33. Ishizuka, J., Yokoyama, A. \& Suemasu, Y. Relationship between serotypes of Bradyrhizobium japonicum and their compatibility with Rj-cultivars for nodulation. Soil Sci. Plant Nutr. 37, 23-30 (1991).

34. Tsurumaru, H., Yamakawa, T., Tanaka, M. \& Sakai, M. Tn5 mutants of Bradyrhizobium japonicum Is-1 with altered compatibility with Rj2-soybean cultivars. Soil Sci. Plant Nutr. 54, 197-203 (2008).

35. Triplett, E. W. \& Sadowsky, M. J. Genetics of competition for nodulation of legumes. Annu. Rev. Microbiol. 46, 399-428 (1992).

36. Sadowsky, M. J., Olson, E. R., Foster, V. E., Kosslak, R. M. \& Verma, D. P. S. Two host-inducible genes of Rhizobium fredii and characterization of the inducing compound. J. Bacteriol. 170, 171-178 (1988).
37. López-Baena, F. J. et al. The absence of Nops secretion in Sinorhizobium fredii HH103 increases GmPR1 expression in Williams soybean. Mol. Plant Microbe Interact. 22, 1445-1454 (2009).

38. Schechter, L. M., Guenther, J., Olcay, E. A., Jang, S. \& Krishnan, H. B. Translocation of NopP by Sinorhizobium fredii USDA257 into Vigna unguiculata root nodules. Appl. Environ. Microbiol. 76, 3758-3761 (2010).

39. Kourelis, J. \& van der Hoorn, R. A. L. Defended to the nines: 25 years of resistance gene cloning identifies nine mechanisms for $\mathrm{R}$ protein function. Plant Cell 30, 285-299 (2018).

40. Zhu, M. et al. The intracellular immune receptor Sw-5b confers broadspectrum resistance to tospoviruses through recognition of a conserved 21amino acid viral effector epitope. Plant Cell 29, 2214-2232 (2017).

41. Salcedo, A. et al. Variation in the AvrSr35 gene determines Sr35 resistance against wheat stem rust race Ug99. Science 358, 1604-1606 (2017).

42. Vlot, A. C., Dempsey, D. A. \& Klessig, D. F. Salicylic acid, a multifaceted hormone to combat disease. Annu. Rev. Phytopathol. 47, 177-206 (2009).

43. Yoshioka, K. et al. Environmentally sensitive, SA-dependent defense responses in thecpr22 mutant of Arabidopsis. Plant J. 26, 447-459 (2001).

44. Yasuda, M. et al. Effector-triggered immunity determines host genotypespecific incompatibility in legume-Rhizobium symbiosis. Plant Cell Physiol. 57, 1791-1800 (2016)

45. Jones, J. D. \& Dangl, J. L. The plant immune system. Nature 444, 323-329 (2006).

46. Anderson, J. P. et al. Plants versus pathogens: an evolutionary arms race. Funct. Plant Biol. 37, 499-512 (2010).

47. Cole, M. A. \& Elkan, G. H. Transmissible resistance to penicillin G, neomycin, and chloramphenicol in Rhizobium japonicum. Antimicrob. Agents Chemother. 4, 248-253 (1973).

48. Miller, J. H. A Short Course in Bacterial Genetics: A Laboratory Manual and Handbook for Escherichia coli and Related Bacteria. (CSHL Press, Cold Spring Harbor, NY., 1992)

49. An, G., Ebert, P. R., Mitra, A. \& Ha, S. B. Binary Vectors., A3:1-19 (Kluer Academic, 1998).

50. Sambrook, J. \& Russell, D. W. Molecular Cloning: A Laboratory Manual, 3 edn (CSHL Press, 2001)

51. Akao, S. \& Kouchi, H. Light microscopic observation of root hair curling of soybean induced by Rhizobium infection. Jpn J. Soil Sci. Plant Nutr. 60, 53-55 (1989).

52. Itakura, M. et al. Generation of Bradyrhizobium japonicum mutants with increased $\mathrm{N}_{2} \mathrm{O}$ reductase activity by selection after introduction of a mutated dnaQ gene. Appl. Environ. Microbiol. 74, 7258-7264 (2008).

53. Ohtsubo, Y., Maruyama, F., Mitsui, H., Nagata, Y. \& Tsuda, M. Complete genome sequence of Acidovorax sp. strain KKS102, a polychlorinatedbiphenyl degrader. J. Bacteriol. 194, 6970-6971 (2012)

54. Sasaki, S., Minamisawa, K. \& Mitsui, H. A Sinorhizobium meliloti RpoHregulated gene is involved in iron-sulfur protein metabolism and effective plant symbiosis under intrinsic iron limitation. J. Bacteriol. 198, 2297-2306 (2016).

55. Kinoshita, E., Kinoshita-Kikuta, E., Takiyama, K. \& Koike, T. Phosphatebinding tag, a new tool to visualize phosphorylated proteins. Mol. Cell. Proteom. 5, 749-757 (2006)

56. Schäfer, A. et al. Small mobilizable multi-purpose cloning vectors derived from the Escherichia coli plasmids pK18 and pK19: selection of defined deletions in the chromosome of Corynebacterium glutamicum. Gene 145, 69-73 (1994)

57. Figurski, D. H. \& Helinski, D. R. Replication of an origin-containing derivative of plasmid RK2 dependent on a plasmid function provided in trans. Proc. Natl Acad. Sci. USA 76, 1648-1652 (1979).

58. Ho, S. N., Hunt, H. D., Horton, R. M., Pullen, J. K. \& Pease, L. R. Site-directed mutagenesis by overlap extension using the polymerase chain-reaction. Gene 77, 51-59 (1989).

59. Kereszt, A. et al. Agrobacterium rhizogenes-mediated transformation of soybean to study root biology. Nat. Protoc. 2, 948-952 (2007).

60. Broughton, W. J. \& Dilworth, M. J. Control of leghaemoglobin synthesis in snake beans. Biochem. J. 125, 1075-1080 (1971).

61. Brechenmacher, L. et al. Transcription profiling of soybean nodulation by Bradyrhizobium japonicum. Mol. Plant Microbe Interact. 21, 631-645 (2008)

62. Thompson, J. D., Higgins, D. G. \& Gibson, T. J. Clustal-W: improving the sensitivity of progressive multiple sequence alignment through sequence weighting, position-specific gap penalties and weight matrix choice. Nucleic Acids Res. 22, 4673-4680 (1994).

63. Kumar, S., Stecher, G. \& Tamura, K. MEGA7: molecular evolutionary genetics analysis version 7.0 for bigger datasets. Mol. Biol. Evol. 33, 1870-1874 (2016).

64. Zehner, S., Schober, G., Wenzel, M., Lang, K. \& Göttfert, M. Expression of the Bradyrhizobium japonicum type III secretion system in legume nodules and analysis of the associated tts box promoter. Mol. Plant Microbe Interact. 21, 1087-1093 (2008). 


\section{Acknowledgements}

We thank Professor Neung Teaumroong (Suranaree University of Technology) for providing mung bean seeds. We thank Dr. Cristina Sánchez and Mr. Shohei Kusakabe (Tohoku University) for advice on mutant construction and protein secretion assay, respectively. We are grateful to Ms. Kaori Kakizaki (Tohoku University) for technical supports in MiSeq sequencing of the spontaneous mutants, and to Mr. Tomoyuki Sakata (Kyushu University) for technical support in DNA sequencing of Is-1 mutants. We thank Dr. Michiko Yasuda (Tokyo University of Agriculture and Technology) and Dr. Yasuyuki Kawaharada (Iwate University) for advice on microscopic observation of soybean roots. This work was supported by JSPS KAKENHI Grant Numbers 26252065 (to K.M.) and 15K20868 (to M.S.) from the Ministry of Education, Culture, Sports, Science and Technology of Japan.

\section{Author contributions}

M.S., H.I., and H.O. carried out screening, resequencing, and genetic validation of USDA 122 spontaneous mutants. M.S. constructed mutant strains and nopP variants of $B$. diazoefficiens. M.S., S.T., Y.S., and H.K. participated in inoculation tests of B. diazoefficiens and its mutants. M.S. and Y.K. carried out gene expression analysis of soybean plants. Y.U. carried out the Rj2/rj2 complementation test. M.S. and S.T. sequenced nopP and constructed the phylogenetic tree. H.T. and T.Y. sequenced nopP in Tn5 mutants of B. diazoefficiens Is-1. M.S., S.S., H.M., and K.M. conceived and coordinated experiments. M.S., Y.U., and K.M. wrote the manuscript.

\section{Additional information}

Supplementary Information accompanies this paper at https://doi.org/10.1038/s41467018-05663-x.
Competing interests: The authors declare no competing interests.

Reprints and permission information is available online at http://npg.nature.com/ reprintsandpermissions/

Publisher's note: Springer Nature remains neutral with regard to jurisdictional claims in published maps and institutional affiliations.

(c) (i)

Open Access This article is licensed under a Creative Commons Attribution 4.0 International License, which permits use, sharing, adaptation, distribution and reproduction in any medium or format, as long as you give appropriate credit to the original author(s) and the source, provide a link to the Creative Commons license, and indicate if changes were made. The images or other third party material in this article are included in the article's Creative Commons license, unless indicated otherwise in a credit line to the material. If material is not included in the article's Creative Commons license and your intended use is not permitted by statutory regulation or exceeds the permitted use, you will need to obtain permission directly from the copyright holder. To view a copy of this license, visit http://creativecommons.org/ licenses/by/4.0/.

(C) The Author(s) 2018 